\title{
DIVISOR SPACES ON PUNCTURED RIEMANN SURFACES
}

\author{
SADOK KALLEL
}

\begin{abstract}
In this paper, we study the topology of spaces of $n$-tuples of positive divisors on (punctured) Riemann surfaces which have no points in common (the divisor spaces). These spaces arise in connection with spaces of based holomorphic maps from Riemann surfaces to complex projective spaces. We find that there are Eilenberg-Moore type spectral sequences converging to their homology. These spectral sequences collapse at the $E^{2}$ term, and we essentially obtain complete homology calculations. We recover for instance results of F. Cohen, R. Cohen, B. Mann and J. Milgram, The topology of rational functions and divisors of surfaces, Acta Math. 166 (1991), 163-221. We also study the homotopy type of certain mapping spaces obtained as a suitable direct limit of the divisor spaces. These mapping spaces, first considered by G. Segal, were studied in a special case by F. Cohen, R. Cohen, B. Mann and J. Milgram, who conjectured that they split. In this paper, we show that the splitting does occur provided we invert the prime two.
\end{abstract}

\section{INTRODUCTION}

Let $X=M_{g}$ be a genus $g$ compact oriented Riemann surface with $g \geq 0$, $M_{0}=\mathbb{P}^{1}$ being the Riemann sphere. For the rest of this paper, we will make use of a preferred basepoint $x_{0}($ or $*)$ in $M_{g}$. Let $S P^{r}(X)$ denote the $r$-fold symmetric product of $X$ (i.e. the space of degree $r$ positive divisors on $X$ ). We define the subspace

$$
\operatorname{Div}_{k_{1}, \ldots, k_{n}}(X) \subset S P^{k_{1}}(X) \times \cdots \times S P^{k_{n}}(X)
$$

to be the set of tuples of positive divisors $\left(D_{k_{1}}, \ldots, D_{k_{n}}\right)$ such that $D_{k_{1}} \cap \cdots \cap D_{k_{n}}=$ $\emptyset$. In other words, $\operatorname{Div}_{k_{1}, \ldots, k_{n}}(X)$ is the space of divisors $D_{k_{i}}$ on $X, i=1, \ldots, n$, of degree $k_{i}$, and having no points in common. The relevance of these spaces of divisors to spaces of holomorphic maps is now explained.

First assume $g=0$. Then by associating to every meromorphic function on $\mathbb{P}^{1}$ its (disjoint) sets of zeros and poles, we can identify the divisor $\operatorname{space}_{\operatorname{Div}}, k\left(S^{2}-*\right)$ with the space of degree $k$ based self-holomorphic maps of the Riemann sphere; that is,

$$
\operatorname{Div}_{k, k}\left(S^{2}-x_{0}\right)=\operatorname{Hol}_{k}^{*}\left(\mathbb{P}^{1}, \mathbb{P}^{1}\right)
$$

Received by the editors December 7, 1995.

1991 Mathematics Subject Classification. Primary 57R19; Secondary 14H55.

Key words and phrases. Riemann surfaces, symmetric products, Eilenberg-Moore spectral sequence, Whitehead product, Milgram bar construction.

The author holds a Postdoctoral fellowship with the Centre de Recherches Mathématiques, Université de Montréal. 
More precisely, the space $\operatorname{Hol}_{k}^{*}\left(\mathbb{P}^{1}, \mathbb{P}^{1}\right)$ here consists of rational maps $f(z)=$ $p(z) / q(z), z \in \mathbb{C}$, where $p$ and $q$ are monic polynomials of degree $k$ (hence some people use the notation $\operatorname{Rat}_{k}$ for $\operatorname{Hol}_{k}^{*}\left(\mathbb{P}^{1}, \mathbb{P}^{1}\right)$ ). We may also write $\operatorname{Rat}_{k}\left(\mathbb{P}^{n}\right)$ for $\operatorname{Hol}_{k}^{*}\left(\mathbb{P}^{1}, \mathbb{P}^{n}\right)$ and here too we have the identification $([\mathrm{S}])$

$$
\operatorname{Div}_{\underbrace{k, k, \ldots, k}_{n+1}}\left(S^{2}-x_{0}\right)=\operatorname{Rat}_{k}\left(\mathbb{P}^{n}\right) .
$$

When $g \geq 1$, the connection between the divisor spaces Div and spaces of holomorphic maps is less direct and is given essentially by a classical theorem of Abel. Recall that every Riemann surface $M_{g}$ embeds, via the Abel-Jacobi map $\mu$ ([ACGH] $)$, into its associated Jacobi variety $J\left(M_{g}\right)$, which is a complex $g$ dimensional torus. The map $\mu$ extends additively to $S P^{k}\left(M_{g}\right), \forall k$. A classical theorem of Abel ([ACGH], chap. I) translates to the statement that the space of degree $k$ based holomorphic maps $\operatorname{Hol}_{k}^{*}\left(M_{g}, \mathbb{P}^{n}\right)$ is the subspace of $\operatorname{Div}_{k, \ldots, k}\left(M_{g}-x_{0}\right)$ consisting of $(n+1)$-tuples of divisors with the property that

$$
\mu\left(D_{1}\right)=\mu\left(D_{2}\right)=\cdots=\mu\left(D_{n+1}\right) .
$$

Using the spaces $\operatorname{Div}\left(M_{g}-x_{0}\right)$ as intermediate constructs, Segal was able to prove an interesting stability result for spaces of holomorphic maps on Riemann surfaces. More explicitly, he showed that the natural inclusion $I: \operatorname{Hol}_{k}^{*}\left(M_{g}, \mathbb{P}^{n}\right) \hookrightarrow$ $\operatorname{Map}_{k}^{*}\left(M_{g}, \mathbb{P}^{n}\right)$, obtained by simply forgetting the holomorphic structure, induces a homotopy equivalence through a range increasing with $k$. These results are greatly extended in $[\mathrm{KM}]$.

A systematic study of the divisor spaces was initiated in [C2M2], where the authors constructed a homotopy model whose cohomology is related to the homology of the Div spaces via Alexander-Poincaré duality. Starting with that model, we are able to construct a homology spectral sequence of the Eilenberg-Moore type, converging to the homology of the Div spaces, and then show that this spectral sequence collapses at the $E^{2}$ term for all $g \geq 0$ and for all $n$. This then yields our first main theorem:

Theorem 0.4. For field coefficients $\mathbb{F}$, we have the following isomorphism:

$$
H_{*}\left(\operatorname{Div}_{\underbrace{k, \ldots, k}_{n}}^{k, \ldots}\left(M_{g}-*\right) ; \mathbb{F}\right) \cong \operatorname{Tor}_{2 n k-*, k}^{H_{*}\left(S P^{\infty}\left(M_{g}\right)\right)}\left(\mathbb{F}, H_{*}\left(S P^{\infty}\left(M_{g}\right) ; \mathbb{F}\right)^{\otimes n}\right) .
$$

To clarify the statement of the theorem above, we need to indicate that there is a bigraded algebra structure on the homology groups of $S P^{\infty}(X)$ yielding in the appropriate manner the bigrading of the Tor term above. The theorem and the details leading to it are discussed in $\S 4$. When $n>2$, the module structure of $H_{*}\left(S P^{\infty}\left(M_{g}\right)\right)^{\otimes n}$ over $H_{*}\left(S P^{\infty}\left(M_{g}\right)\right)$ is trivial and so the calculations are direct. We write $\operatorname{Div}_{\underbrace{}_{n}, \ldots, k}^{k, \ldots}=\operatorname{Div}_{k}^{n}$. One has for instance $(\S 6)$

Corollary 0.5. For $n>2$ and $g \geq 1$, the rational homology of $\operatorname{Div}_{k}^{n}\left(M_{g}-*\right)$ is the subset of the $(n+1)$-graded algebra

$$
\Lambda\left(e_{1 ; 1}, \ldots, e_{2 g ; 1}, \ldots, e_{1 ; n}, \ldots, e_{2 g ; n}, E\right) \otimes \mathbb{Q}\left(h_{1}, \ldots, h_{2 g}\right),
$$

where the grading is assigned as follows: $e_{i ; r} \mapsto(1 ; 0, \ldots, 1, \ldots, 0)$, with 1 in the $r+1$ position, $1 \leq r \leq n, 1 \leq i \leq 2 g, E \mapsto(2 n-3 ; 1, \ldots, 1), h_{j} \mapsto(2 n-2 ; 1, \ldots, 1)$. 
The multigrading is additive. In this setting, $H_{*}\left(\operatorname{Div}_{k}^{n}\left(M_{g}-*\right) ; \mathbb{Q}\right)$ is given by those elements of multidegree $\left(* ; i_{1}, \ldots, i_{n}\right)$ with $i_{j} \leq k$.

Similar results are obtained $\bmod -p$. When $g=0,0.4$ takes a quite simple and explicit expression for all $n$, and one recovers the original results of [C2M2] on the homology structure of the Rat spaces ( 55 ). In the case when $n=2$, the module structure in the Tor term of 0.4 is non-trivial and the calculations are much more tedious.

Remark 0.6. The spectral sequence that is considered in this paper and the resulting collapse are used in $[\mathrm{KM}]$ to study the spaces $\operatorname{Hol}_{k}^{*}\left(M_{g}, \mathbb{P}^{n-1}\right)$ themselves. It's not coincidental that there too the homology structure does depend on whether $n=2$ or $n>2$.

We can stabilize the divisor spaces with respect to "collar" inclusions

$$
\operatorname{Div}_{k_{1}, \ldots, k_{n}}\left(M_{g}-*\right) \rightarrow \operatorname{Div}_{k_{1}, \ldots, k_{j-1}, k_{j}+1, k_{j+1}, \ldots, k_{n}}\left(M_{g}-*\right)
$$

obtained by first continuously deforming $\operatorname{Div}_{k_{1}, \ldots, k_{n}}\left(M_{g}-*\right)$ to $\operatorname{Div}_{k_{1}, \ldots, k_{n}}\left(M_{g}-U\right)$ where $U$ is a small neighborhood of $*$, and then adding a chosen point $x \neq * \in U$ to the $j$ th divisor. It is now a theorem of Segal that the direct limit over these inclusions is homotopy equivalent to a component of a known (based) mapping space; i.e.

$$
\lim _{k \rightarrow \infty} \operatorname{Div}_{k}^{n}\left(M_{g}-*\right) \simeq \operatorname{Map}_{0}^{*}\left(M_{g}, W_{n}(\mathbb{P})\right) \quad(\text { Segal })
$$

where $W_{n}(\mathbb{P})$ is the $n^{t h}$ fat wedge of the infinite complex projective space $\mathbb{P}$ (or $\mathbb{P}^{\infty}$ ) and where $\mathrm{Map}_{0}$ denotes the component of null-homotopic maps. The fat wedge $W_{n}(X) \subset X^{n}$ is the subset consisting of tuples where at least one entry is basepoint (e.g. $W_{1}=* \in X$ and $\left.W_{2}=X \vee X\right)$. In $\S 7$ we establish the existence of a fibration with a section

$$
S^{2 n-1} \rightarrow W_{n}\left(\mathbb{P}^{\infty}\right) \hookrightarrow\left(\mathbb{P}^{\infty}\right)^{n}
$$

which when coupled with the mapping space fibration obtained by mapping the cofibration sequence $\bigvee S^{1} \rightarrow M_{g} \rightarrow S^{2}$ into $W_{n} \mathbb{P}$, yields the fibration

$$
\Omega^{2} S^{2 n-1} \rightarrow \operatorname{Map}_{0}^{*}\left(M_{g}, W_{n} \mathbb{P}\right) \rightarrow\left(S^{1}\right)^{2 n g} \times\left(\Omega S^{2 n-1}\right)^{2 g} .
$$

It is now not hard to see $(\S 9)$ that as a result of 0.4 and 0.7 we have

Proposition 0.9. The (cohomology) Eilenberg-Moore spectral sequence associated to

$$
\Omega^{2} S^{2 n-1} \rightarrow \operatorname{Map}_{0}^{*}\left(M_{g}, W_{n} \mathbb{P}\right) \rightarrow\left(\Omega W_{n} \mathbb{P}\right)^{2 g}
$$

and converging to $H^{*}\left(\operatorname{Map}_{0}^{*}\left(M_{g}, W_{n} \mathbb{P}\right) ; \mathbb{F}\right)$ collapses at the $E_{2}$ term.

This leads to the determination of $H^{*}\left(\operatorname{Map}_{0}^{*}\left(M_{g}, W_{n} \mathbb{P}\right) ; \mathbb{F}\right)$, and the results turn out to be consistent with the conjecture of [C2M2] which states that the term $\left(\Omega S^{2 n-1}\right)^{2 g}$ in the base of 0.8 ought to split off from the mapping space. More explicitly, and in the relevant case when $n=2$, [C2M2] states that there should be a decomposition

$$
\operatorname{Map}^{*}\left(M_{g}, \mathbb{P} \vee \mathbb{P}\right) \simeq(\mathbb{Z})^{2} \times \Omega\left(S^{3}\right)^{2 g} \times Y_{g}
$$

where $Y_{g}$ is the total space of a fibration $\Omega^{2}\left(S^{3}\right) \rightarrow Y_{g} \rightarrow\left(S^{1}\right)^{4 g}$. The existence of such a splitting is also very much suggested by results of [BCM], who prove similar 
decomposition results for $\operatorname{Map}^{*}\left(M_{g}, S^{2 n}\right), n \geq 1$ (see $\S 7$ ). It turns out, however, that there is an obstruction to such a decomposition.

In $\S 8$ we study the homotopy type of the mapping space $\operatorname{Map}^{*}\left(M_{g}, W_{n}(\mathbb{P})\right)$. The problem there becomes to factor the classifying map associated to 0.8 as

$$
f^{!}:\left(\Omega S^{2 n+1} \times\left(S^{1}\right)^{n+1}\right)^{2 g} \rightarrow\left(S^{1}\right)^{2 g(n+1)} \rightarrow \Omega S^{2 n+1} \hookrightarrow \Omega S^{2 n+1} \times\left(S^{1}\right)^{(n+1)} .
$$

A first look at $f^{!}$shows that there are essential $\mathbb{Z}_{2}$ obstructions to such a factorization when $n>2$. When $n=2$, a close examination of the Postnikov system of $\mathbb{P}^{\infty} \vee \mathbb{P}^{\infty}$ shows that there is a non-zero obstruction to the above decomposition taking the form of a triple Whitehead product

$$
\left[a_{1},\left[a_{1}, a_{2}\right]\right] \in \pi_{4}(\mathbb{P} \vee \mathbb{P}) \cong \mathbb{Z}_{2} .
$$

So in all cases we're up against essential $\mathbb{Z}_{2}$ obstructions, and we have

Proposition 0.10. There is a splitting after inverting 2 :

$$
\operatorname{Map}_{0}^{*}\left(M_{g}, W_{n} \mathbb{P}\right) \simeq\left(\Omega S^{2 n-1}\right)^{2 g} \times Y_{g, n},
$$

where $Y_{g, n}$ is the total space of a fibration $\Omega^{2}\left(S^{2 n-1}\right) \rightarrow Y_{g, n} \rightarrow\left(S^{1}\right)^{2 n g}$.

\section{The Structure of Symmetric Products}

Given a space $X$, we let $S P^{n}(X)=X^{n} / \mathcal{S}_{n}$ denote the $n$-th symmetric product of $X$ (here $\mathcal{S}_{n}$ is the group on $n$ letters acting by permuting factors). Equivalently, $S P^{n}(X)$ is the set of all unordered $n$-tuples $\left\langle x_{1}, \ldots, x_{n}\right\rangle$ of points in $X$.

Let $*$ be a chosen base point in $X$; then there are natural inclusions $S P^{n}(X) \hookrightarrow$ $S P^{n+1}(X)$ which identify $\left\langle x_{1}, \ldots, x_{n}\right\rangle$ with $\left\langle x_{1}, \ldots, x_{n}, *\right\rangle$, and we get the expanding sequence of spaces

$$
* \equiv S P^{0}(X) \subset S P^{1}(X) \subset \cdots \subset S P^{n-1}(X) \subset S P^{n}(X) \subset \cdots .
$$

The direct limit over these inclusions is the infinite symmetric product $S P^{\infty}(X, *)$ (topologized by the weak topology relative to the union of the $S P^{i}(X)$.) The pairing

$$
\begin{array}{ccc}
S P^{n}(X) \times S P^{m}(X) & \stackrel{\mu}{\rightarrow} & S P^{n+m}(X), \\
\left\langle x_{1}, \ldots, x_{n}\right\rangle \times\left\langle y_{1}, \ldots, y_{m}\right\rangle & \mapsto & \left\langle x_{1}, \ldots, x_{n}, y_{1}, \ldots, y_{m}\right\rangle,
\end{array}
$$

turns $S P^{\infty}(X, *)$ into an abelian and associative monoid with pairing

$$
\mu: S P^{\infty}(X, *) \times S P^{\infty}(X, *) \rightarrow S P^{\infty}(X, *) .
$$

We often write $($ or +$)$ for addition in $S P^{\infty}(X, *)$; that is, $\mu((x, y))=x \cdot y=$ $x+y=x y$ are all equivalent notations. We use the same notation for the induced Pontryagin product on $H_{*}\left(S P^{\infty}(X) ; \mathbb{A}\right)$.

Since $S P^{\infty}(X, *)$ is abelian, it must be a product of Eilenberg-Mac Lane spaces, and one actually has

Theorem 1.1 (Dold-Thom). $\pi_{*}\left(S P^{\infty}(X, *)\right) \cong H_{*}(X ; \mathbb{Z})$, and hence

$$
S P^{\infty}(X, *)=\prod K\left(\tilde{H}_{i}(X ; \mathbb{Z}), i\right) .
$$

For example, $S P^{\infty}\left(S^{n}, *\right) \simeq K(\mathbb{Z}, n), n \geq 1$.

Properties. - The finite and infinite symmetric products are covariant functors on the category of pointed topological spaces. If $f:(X, *) \rightarrow(Y, *)$ is a map of pairs, then the induced maps on the symmetric products are denoted by $S P^{n} f$ : $S P^{n}(X) \rightarrow S P^{n}(Y)$ and $S P^{\infty} f: S P^{\infty}(X, *) \rightarrow S P^{\infty}(Y, *)$. 
- $S P^{n}(-)$ is a homotopy functor. In particular, if $c X$ denotes the cone on $X$, then $S P^{n}(c X)$ is contractible for all $n$.

Facts. Here are now some known properties of symmetric products that we will be using:

- $S P^{\infty}(X \vee Y, *) \simeq S P^{\infty}(X, *) \times S P^{\infty}(Y, *)$.

- $\pi_{1}\left(S P^{n}(X)\right)$ is abelian when $n \geq 2$.

- $S P^{n}\left(S^{1}\right) \simeq S^{1}, n \geq 1$.

- $S P^{n}\left(S^{2}\right) \cong \mathbb{P}^{n}, n \geq 1$.

- There is a diffeomorphism $S P^{n}(\mathbb{C}) \cong \mathbb{C}^{n}$.

To see this last statement, choose a tuple of $n$ points in $\mathbb{C}$, say $\left(v_{1}, v_{2}, \ldots, v_{n}\right)$, and associate to it the coefficients of the monic polynomial $\left(z-v_{1}\right)\left(z-v_{2}\right) \cdots\left(z-v_{n}\right)$. This sets up the correspondence between $S P^{n}(\mathbb{C})$ and $\mathbb{C}^{n}$, and it's easy to see that it is a diffeomorphism. A straightforward corollary of this is:

Corollary 1.5. Let $M$ be a smooth closed curve. Then $S P^{n}(M)$ is a complex $n$ dimensional manifold.

1.1. The Homology of Symmetric Products. The symmetric products exhibit interesting homological properties. For instance, it was proved by Dold [D] that for $X$ a CW-complex, $H_{*}\left(X^{n} / G\right)$ only depends on $H_{*}(X)$ for any subgroup $G \subset \mathcal{S}_{n}$. The homology groups $H_{*}\left(S P^{n}(X)\right)$, for instance, are entirely determined by the homology groups of $X$. Moreover, we have the following classical splitting result due to Steenrod:

Theorem 1.6 (Steenrod). For $X$ connected and for untwisted coefficients $\mathbb{A}$, we have

$$
\begin{aligned}
H_{*}\left(S P^{n}(X) ; \mathbb{A}\right) & =\sum_{k=1}^{n} H_{*}\left(S P^{k}(X), S P^{k-1}(X) ; \mathbb{A}\right) \\
& =H_{*}\left(S P^{n}(X), S P^{n-1}(X) ; \mathbb{A}\right) \oplus H_{*}\left(S P^{n-1}(X) ; \mathbb{A}\right) .
\end{aligned}
$$

Remark 1.7. The splitting above induces a bigrading on $H_{*}\left(S P^{\infty}(X, *), \mathbb{A}\right)$; for an element $x \in H_{*}\left(S P^{\infty}(X, *), \mathbb{A}\right)$ has bidegree $(i, k)$ iff

$$
x \in H_{i}\left(S P^{k}(X), S P^{k-1}(X), \mathbb{A}\right) .
$$

This evidently implies that $H_{*}\left(S P^{\infty}(X, *), \mathbb{A}\right)$ has the structure of a bigraded algebra. We will write $\operatorname{deg}(x)$ for the homological degree of $x$ and fil $(x)$ for its filtration degree $k$. Notice that

$$
\operatorname{deg}(x \cdot y)=\operatorname{deg}(x)+\operatorname{deg}(y), \operatorname{fil}(x \cdot y)=\operatorname{fil}(x)+\operatorname{fil}(y) .
$$

Remark 1.8. For finitely generated $\mathrm{CW}$-complexes, there is a standard procedure due to Milgram [M3] to determine the homology of the symmetric products. This procedure amounts to first determining the bigraded algebra structure of

$$
H_{*}\left(S P^{\infty} A(G, n) ; \mathbb{A}\right)=H_{*}(K(G, n), \mathbb{A})
$$

for Moore spaces, and this can be deduced from Cartan's determination of the homology of Eilenberg-Mac Lane spaces [Car].

Knowledge of the homology of symmetric products of Moore spaces can then be used to determine $H_{*}\left(S P^{\infty}(X, *)\right)$ for any finitely generated $\mathrm{CW}$-complex $X$. More precisely, given such $X$ (arcwise connected), one can recover the homology type of $X$ via a wedge of Moore spaces $Y_{i}$, and hence the problem reduces to calculating 
$H_{*}\left(S P^{\infty}\left(\bigvee Y_{i}, *\right)\right)$ as a bigraded algebra. But it's not hard to see that for CWcomplexes $X$ and $Y$ (and untwisted coefficients $\mathbb{A}$ ) there is a bigraded algebra isomorphism

$$
H_{*}\left(S P^{\infty}(X \vee Y, *), \mathbb{A}\right) \cong H_{*}\left(S P^{\infty}(X, *), \mathbb{A}\right) \otimes H_{*}\left(S P^{\infty}(Y, *), \mathbb{A}\right) .
$$

1.2. Symmetric Products of Curves. A genus $g$ compact Riemann surface $M_{g}$ is obtained by attaching a 2-cell, $D^{2}$, to a wedge of $2 g$-circles via the commutator map. If we denote by $a_{1}, b_{1}, \cdots, a_{g}, b_{g}$ the generators of $\pi_{1}\left(M_{g}\right)$, each representing a copy of $S^{1}$ in the one skeleton $\underbrace{S^{1} \vee \cdots \vee S^{1}}_{2 g} \subset M_{g}$, then we can write $M_{g} \simeq$ $\left(\bigvee^{2 g} S^{1}\right) \cup_{\left[a_{1}, b_{1}\right] \cdots\left[a_{g}, b_{g}\right]} D^{2}$

We choose the letters $\left\{e_{i}, i=1, \ldots, 2 g\right\}$ to label the homology generators in $H_{1}\left(M_{g} ; \mathbb{Z}\right)$. The boundary of the top 2-dimensional class $D^{2}$ vanishes (being a commutator), and hence $D^{2}$ generates a homology class which corresponds to the orientation class $\left[M_{g}\right]$ (or $M$ for short). In homology we have that $H_{*}\left(M_{g}\right) \cong$ $H_{*}\left(\bigvee^{2 g}\left(S^{1}\right) \vee S^{2}\right)$ and it follows from 1.1 and 1.8 that

Lemma 1.9. We have the following bigraded algebra isomorphism:

$$
H_{*}\left(S P^{\infty}\left(M_{g}, *\right) ; \mathbb{Z}\right) \cong \Lambda\left(e_{1}\right) \otimes \cdots \otimes \Lambda\left(e_{2 g}\right) \otimes \Gamma[M]
$$

where $\Gamma[M]$ is the divided power algebra over $\mathbb{Z}$ generated by elements $\gamma_{i}=\frac{M^{i}}{i !}$.

Here it is clear that $\operatorname{deg}\left(e_{i}\right)=1=\operatorname{fil}\left(e_{i}\right)$ and so the $e_{i}$ 's have bidegree $(1,1)$, while $M$ has bidegree $(2,1)$. As a consequence of 1.6 one can check that

Lemma 1.10. $H_{*}\left(S P^{n}\left(M_{g}\right) ; \mathbb{Z}\right) \subset H_{*}\left(S P^{\infty}\left(M_{g}, *\right) ; \mathbb{Z}\right)$ consists of all elements of bidegree $(*, i), i \leq n$. For instance $H_{*}\left(S P^{n}(M), S P^{n-1}(M) ; \mathbb{Z}\right)$ has generators of the following type:

$$
e_{i_{1}} \cdots e_{i_{r}} \gamma_{s}, r+s=n .
$$

Lemma 1.10 describes entirely the homology of $S P^{n}\left(M_{g}\right)$ for finite $n$. Notice at this point that 1.1 and then 1.3 imply that

$$
S P^{\infty}\left(M_{g}, *\right) \simeq K\left(\mathbb{Z}^{2 g}, 1\right) \times K(\mathbb{Z}, 2) \simeq\left(S^{1}\right)^{2 g} \times \mathbb{P} \simeq S P^{\infty}\left(S^{1}\right)^{2 g} \times S P^{\infty}\left(S^{2}\right) .
$$

We can give an explicit construction of the homotopy equivalence above as follows. First we have the obvious map $S P^{\infty}\left(\bigvee^{2 g} S^{1}, *\right)=S P^{\infty}\left(S^{1}, *\right)^{2 g} \stackrel{S P^{\infty}}{\rightarrow}(i)$ $S P^{\infty}\left(M_{g}, *\right)$ induced from the inclusion of the one skeleton $i: \bigvee^{2 g} S^{1} \hookrightarrow M_{g}$ and sending the wedgepoint to basepoint $* \in M_{g}$. Next, we can consider the composite

$$
\tau: S^{1}=\partial D^{2} \rightarrow \bigvee^{2 g} S^{1} \rightarrow S P^{2}\left(\bigvee^{2 g} S^{1}\right) \rightarrow S P^{2}\left(M_{g}\right)
$$

At the level of fundamental groups, $\tau_{*}$ factors through a commutator $f_{*}$ and since $\pi_{1}\left(S P^{2}(X)\right)$ is abelian $(1.2)$, it follows that $\tau_{*}\left(\left[S^{1}\right]\right)=0$. The map $\tau$ extends to a map from a new disk $D^{\prime 2}$,

$$
\tau: D^{\prime 2} \rightarrow S P^{2}\left(\bigvee^{2 g} S^{1}\right) \rightarrow S P^{2} M_{g}
$$


We can draw the following diagram:

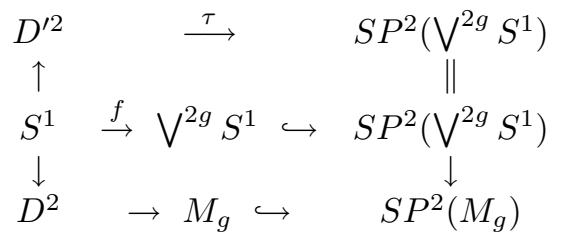

which is then seen to give rise to a map

$$
h: S^{2}=D^{2} \cup_{S^{1}} D^{\prime 2} \rightarrow S P^{2}\left(M_{g}\right)
$$

and hence to a map $S P^{\infty}(h): S P^{\infty}\left(S^{2}, *\right)=\mathbb{P}^{\infty} \rightarrow S P^{\infty}\left(M_{g}, *\right)$.

Lemma 1.13. The composite

$$
\mu\left(S P^{\infty}(i) \times S P^{\infty}(h)\right):\left(S P^{\infty}\left(S^{1}, *\right)\right)^{2 g} \times S P^{\infty}\left(S^{2}, *\right) \rightarrow S P^{\infty}\left(M_{g}, *\right)
$$

is a homotopy equivalence (here again $\mu$ is the monoid addition in $S P^{\infty}\left(M_{g}, *\right)$ ).

About divided power algebras. Let $A$ be a commutative graded algebra, and $a \in A$ an even degree element. A divided power algebra on $a$; denoted by $\Gamma(a)$, is the algebra generated by elements $\gamma_{i}=\gamma_{i}(a)$ with relations

$$
\gamma_{0}=1, \gamma_{1}=a, \gamma_{k} \gamma_{h}=\left(\begin{array}{c}
k+h \\
h
\end{array}\right) \gamma_{k+h},
$$

and boundary $d \gamma_{k}=(d a) \gamma_{k-1}, k \geq 1$. Here the degree of $\gamma_{k}$ is determined by the fact that $\operatorname{deg}\left(\gamma_{k}\right)=k \operatorname{deg}(a)$. Over $\mathbb{Z}$, the generators of $\Gamma(a)$ are uniquely defined by the formula $\gamma_{k}=\frac{a^{k}}{k !}$. Over $\mathbb{Q}$, everything becomes a unit and hence $\Gamma(a)=\mathbb{Q}[a]$. With mod- $p$ coefficients, $\Gamma(a)$ splits into products of truncated polynomial algebras (see $\S 6)$.

\section{A Model for the Divisor Spaces}

For any space $X$, and given integers $k_{i} \geq 1$, we defined ( $(0)$ the divisor space $\operatorname{Div}_{k_{1}, \ldots, k_{n}}(X)=\left\{\left(D_{k_{1}}, \ldots, D_{k_{n}}\right) \mid D_{k_{i}} \in S P^{k_{i}}(X), D_{k_{1}} \cap D_{k_{2}} \cap \cdots \cap D_{k_{n}}=\emptyset\right\}$.

The element $D_{k} \in S P^{k}(X)$ can be represented either by an unordered $k$-tuple of points $\left\langle x_{1}, \ldots, x_{k}\right\rangle$ (the $x_{j}$ not necessarily distinct), or by a formal sum $\sum n_{i} x_{i}$ such that $\sum n_{i}=k$ and $x_{i} \neq x_{j}$ (when $X$ is a curve, these are called positive divisors in the language of algebraic geometry.)

Notation. We write $\operatorname{Div}_{k_{1}, \ldots, k_{n}}=\operatorname{Div}_{k_{1}, \ldots, k_{n}}\left(M_{g}-*\right)$ and $\operatorname{Div}_{k}^{n}\left(M_{g}-*\right)=$ Div $^{k, \ldots, k}$.

Let $\stackrel{n}{\Delta}$ denote the diagonal multiplication

$$
\begin{gathered}
\left(\coprod_{j=1}^{\infty} S P^{j}(X)\right) \times S P^{k_{1}}(X) \times \cdots \times S P^{k_{n}}(X) \\
\rightarrow \coprod_{j} S P^{k_{1}+j}(X) \times \cdots \times S P^{k_{n}+j}(X)
\end{gathered}
$$

given on points by $\Delta\left(D, D_{1}, \ldots, D_{n}\right)=\left(D_{1}+D, \ldots, D_{n}+D\right)$. It is clear that the divisor spaces $\operatorname{Div}_{k_{1}, \ldots, k_{n}}(X)$ are included in the product $S P^{k_{1}}(X) \times \cdots \times S P^{k_{n}}(X)$ as the complement of $\operatorname{Im}(\Delta)$; that is 
Lemma 2.2. $\operatorname{Div}_{k_{1}, \ldots, k_{n}}=S P^{k_{1}}\left(M_{g}-*\right) \times \cdots \times S P^{k_{n}}\left(M_{g}-*\right)-\operatorname{Im}(\Delta)$.

Remark 2.3. The inclusion $\operatorname{Div}_{k_{1}, \ldots, k_{n}}(X) \subset S P^{k_{1}}(X) \times \cdots \times S P^{k_{n}}(X)$ is an open embedding, and since for curves $X$ the left hand side is a $k_{1}+\ldots+k_{n}$ complex manifold (Corollary 1.5), it follows that $\operatorname{Div}_{k_{1}, \ldots, k_{n}}(X)$ is also a complex manifold of dimension $k_{1}+\ldots+k_{n}$.

We can define at this point the quotient space

$$
\begin{aligned}
T Y_{k_{1}, \ldots, k_{n}}= & S P^{k_{1}}\left(M_{g}\right) \times \cdots \times S P^{k_{n}}\left(M_{g}\right) \\
& /\left\{\bigcup_{i} S P^{k_{1}}\left(M_{g}\right) \times \cdots \times S P^{k_{i}-1}\left(M_{g}\right) \times \cdots \times S P^{k_{n}}\left(M_{g}\right) \cup \operatorname{Im}(\Delta)\right\}
\end{aligned}
$$

which can be thought of as $S P^{k_{1}}\left(M_{g}\right) \times \cdots \times S P^{k_{n}}\left(M_{g}\right) / V$, where $V$ is such that $\operatorname{Div}_{k_{1}, \ldots, k_{n}}=S P^{k_{1}}\left(M_{g}\right) \times \cdots \times S P^{k_{n}}\left(M_{g}\right)-V$. One can therefore invoke PoincaréAlexander duality to write

$$
H^{i}\left(\operatorname{Div}_{k_{1}, \ldots, k_{n}} ; \mathbb{F}\right)=H_{2\left(k_{1}+\cdots+k_{n}\right)-i}\left(T Y_{k_{1}, \ldots, k_{n}} ; \mathbb{F}\right) .
$$

The homology of the quotient space $T Y_{k_{1}, \ldots, k_{n}}$ is not easy to extract when the space is presented in this form. However, we can use a homotopy equivalent construction due to [C2M2] which makes the homological structure much more apparent.

Consider the twisted product space

$$
D Y^{n}(X)=(\underbrace{S P^{\infty}(X, *) \times \cdots \times S P^{\infty}(X, *)}_{n}) \times_{t} S P^{\infty}(c X),
$$

where $t$ identifies the points

$$
\begin{aligned}
\left(D_{1}, \cdots, D_{n},\left\langle\left(t_{1}, z_{1}\right) \cdots\left(t_{k}, z_{k}\right)\right\rangle\right) & \\
& \sim\left(D_{1}+z_{i}, \ldots, D_{n}+z_{i},\left\langle\left(t_{1}, z_{1}\right) \cdots\left(t_{i-1}, z_{i-1}\right)\left(t_{i+1}, z_{i+1}\right) \cdots\left(t_{k}, z_{k}\right)\right\rangle\right)
\end{aligned}
$$

whenever $t_{i}=0$ (here $D_{i} \in S P^{k_{i}}(X)$ for some $k_{i} \geq 1$ and $\left(t_{j}, z_{j}\right) \in c X, t_{j} \in[0,1]$.)

The space $D Y^{n}(X)$ is naturally filtered as follows

$$
D Y_{k_{1}, \ldots, k_{n}}(X)=\bigcup_{\substack{i_{1}+l \leq k_{1} \\ \vdots \\ i_{n}+i \leq k_{n}}}\left(S P^{i_{1}}(X) \times \cdots \times S P^{i_{n}}(X)\right) \times_{t} S P^{l}(c X) .
$$

We observe that there is a projection

$$
p: D Y_{k_{1}, \ldots, k_{n}} \rightarrow S P^{k_{1}}(X) \times \cdots \times S P^{k_{n}}(X) / \operatorname{Im}(\Delta)
$$

given by

$$
\left(D_{i_{1}}, D_{i_{2}}, \ldots, D_{i_{n}},\left\langle\left(t_{1}, z_{1}\right) \cdots\left(t_{l}, z_{l}\right)\right\rangle\right) \mapsto\left(D_{i_{1}}, D_{i_{2}}, \ldots, D_{i_{n}}\right) .
$$

It is easy to see that $p$ is acyclic, inverse images of points being contractible sets. It follows that $p$ induces an isomorphism in homology and combining this with 2.4 yields

Lemma 2.7. There is an isomorphism $H^{i}\left(\operatorname{Div}_{k_{1}, \ldots, k_{n}}\left(M_{g}-*\right) ; \mathbb{F}\right)=H_{2\left(k_{1}+\cdots+k_{n}\right)-i}\left(D Y_{k_{1}, \ldots, k_{n}} / \bigcup_{i} D Y_{k_{1}, \ldots, k_{i}-1, \ldots, k_{n}} ; \mathbb{F}\right)$. 
It is the quotient spaces $D Y_{k_{1}, \ldots, k_{n}} / \bigcup_{i} D Y_{k_{1}, \ldots, k_{i}-1, \ldots, k_{n}}$ that we analyze in this paper.

2.1. Homotopy invariance. Here we show that the topology of the space $D Y$ doesn't depend on the choice of the diagonal approximation $\Delta$. Choose a map $\Delta^{\prime}: S P^{\infty}(X) \rightarrow S P^{\infty}(X)^{n}$ homotopic to $\Delta$ and define the corresponding space $D Y^{\prime}(X)$ obtained from $\left(S P^{\infty}(X, *)\right)^{n} \times S P^{\infty}(c X)$ via the identification

$$
\begin{aligned}
& \left(D_{1}, \cdots, D_{n},\left\langle\left(t_{1}, z_{1}\right) \cdots\left(t_{k}, m_{k}\right)\right\rangle\right) \\
& \quad \sim\left(\nu\left(\Delta^{\prime}\left(z_{i}\right),\left(D_{1}, \cdots, D_{n}\right)\right),\left\langle\left(t_{1}, z_{1}\right) \cdots\left(t_{i-1}, z_{i-1}\right)\left(t_{i+1}, z_{i+1}\right) \cdots\left(t_{k}, m_{k}\right)\right\rangle\right)
\end{aligned}
$$

whenever $t_{i}=0$. Here $\nu$ is the componentwise symmetric product multiplication.

Lemma 2.8. $D Y^{\prime}\left(M_{g}\right) \simeq D Y\left(M_{g}\right)$.

Proof. Denote by $A G(X)$ the free abelian group on points of $X$ or equivalently the group completion of $S P^{\infty}(X)$. Points of $A G(X)$ have the form * or

$$
\left\{x_{1} \cdots x_{r}, y_{1}^{-1} \cdots y_{s}^{-1} \mid * \neq x_{i}, y_{j}, x_{i} \neq y_{j}\right\} \text {. }
$$

It is known ([DT]) that for connected CW complexes, the inclusion $S P^{\infty}(X) \hookrightarrow$ $A G(X)$ is a homotopy equivalence.

For simplicity of notation, write $G=A G\left(M_{g}\right)$. The diagonal $\Delta$ (resp. $\left.\Delta^{\prime}\right)$ extends in the obvious way to a map $G \rightarrow G^{n}$ and it induces an action $\delta: G \times$ $G^{n} \rightarrow G^{n}$ (resp. $\delta^{\prime}$ ) as described previously. We can then consider the associated "completed" model

$$
\hat{D Y}\left(M_{g}\right)=G^{n} \times_{G} A G\left(c M_{g}\right)
$$

where $G$ acts on $G^{n}$ via $\delta$. Similary we can construct $\hat{D Y^{\prime}}\left(M_{g}\right)$ associated to $\delta^{\prime}$. It is easy to see that the new model $\hat{D Y}\left(M_{g}\right)$ is homotopy equivalent to $D Y\left(M_{g}\right)$. This follows by considering the map of quasifiberings

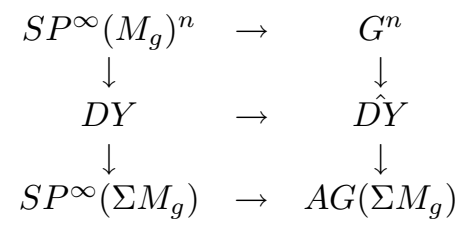

where the top and bottom maps are homotopy equivalences (similarly $\hat{D Y^{\prime}}\left(M_{g}\right) \simeq$ $\left.D Y^{\prime}\left(M_{g}\right)\right)$.

Since $\Delta$ is a diagonal approximation, we have a homotopy $G \times G^{n} \times I \stackrel{\phi}{\rightarrow} G^{n}$, where if we write $\phi(g, x, t)=g_{t}(x)$, the map $g_{0}$ corresponds to componentwise multiplication $G \times G^{n} \rightarrow G^{n}$ and $g_{1}=\delta$. The inclusion $i:\left(G^{n} \times\{0\}\right) \times A G\left(c M_{g}\right) \hookrightarrow$ $\left(G^{n} \times I\right) \times A G\left(c M_{g}\right)$ is a $G$-map (here the action of $G$ on the right hand side is given by $\left.g((x, t), w)=\left(\left(g_{t}(x), t\right), g w\right)\right)$, and it is clearly a homotopy equivalence. Since $G$ acts freely on $A G\left(c M_{g}\right), i$ is a map of free $G$-spaces, and it is then a theorem of equivariant homotopy $[\mathrm{Br}]$ that $i$ is actually a homotopy equivalence through $G$-maps. This then descends to an equivalence of quotients, and we have

$$
\hat{D} Y\left(M_{g}\right)=\left(G^{n} \times\{1\}\right) \times_{G} A G\left(c M_{g}\right) \simeq\left(G^{n} \times\{0\}\right) \times_{G} A G\left(c M_{g}\right) .
$$

The same argument shows that $\hat{D} Y^{\prime}\left(M_{g}\right)$ is homotopy equivalent to the right-hand, side and the lemma is proved. 


\section{Stabilization}

As indicated in the introduction, there are homotopy inclusions

$$
\operatorname{Div}_{k}^{n}=\operatorname{Div}_{k, \ldots, k}\left(M_{g}-*\right) \hookrightarrow \operatorname{Div}_{k+1}^{n}
$$

defined as follows (and more generally, there are inclusions

$$
\operatorname{Div}_{k_{1}, \ldots, k_{i}, \ldots, k_{n}} \hookrightarrow \operatorname{Div}_{k_{1}, \ldots, k_{i}+1, \ldots, k_{n}}
$$

that raise any degree): Choose a sequence of concentric neighborhoods $\left\{U_{k}\right\}, k \geq 1$, $U_{k+1} \subset U_{k}$, around the basepoint $x_{0} \in M_{g}$. For each neighborhood $U_{k}$ pick an $n$ tuple of distinct points $\left(x_{1}^{k}, \ldots, x_{n}^{k}\right) \in U_{k}-U_{k+1}$. The map

$$
\operatorname{Div}_{k}^{n}\left(M_{g}-U_{k}\right) \rightarrow \operatorname{Div}_{k+1}^{n}\left(M_{g}-U_{k+1}\right)
$$

given by sending a configuration $\left(D_{1}, \ldots, D_{n}\right)$ to $\left(D_{1}+x_{1}^{k}, \ldots, D_{n}+x_{n}^{k}\right)$ is a closed embedding, and it extends to an open embedding

$$
e: \operatorname{Div}_{k}^{n}\left(M_{g}-U_{k}\right) \times\left(U_{k}-\bar{U}_{k+1}\right) \rightarrow \operatorname{Div}_{k+1}^{n}\left(M_{g}-U_{k+1}\right) .
$$

It is not hard to see that $\operatorname{Div}_{k}^{n}\left(M_{g}-U_{k}\right) \cong \operatorname{Div}_{k}^{n}$, and so we regard $\operatorname{Div}_{k}^{n}$ as a codimension $2 n$ (real) submanifold of $\operatorname{Div}_{k+1}^{n}$.

Notation. The direct limit of $\operatorname{Div}_{k}^{n}$ over the embeddings $e$ is denoted by $\operatorname{Div}^{n}\left(M_{g}-*\right)$. Consider now the following diagram:

$$
\begin{array}{ccc}
H_{*}\left(\operatorname{Div}_{k}^{n}\right) & \stackrel{e_{*}}{\rightarrow} & H_{*}\left(\operatorname{Div}_{k+1}^{n}\right) \\
\downarrow \cong & & \downarrow \cong \\
H^{2 k n-*}\left(\operatorname{Div}_{k}^{n}, \partial \operatorname{Div}_{k}^{n}\right) & \stackrel{f}{\rightarrow} & H^{2(k+1) n-*}\left(\operatorname{Div}_{k+1}^{n}, \partial \operatorname{Div}_{k+1}^{n}\right) \\
\downarrow \cong & & \downarrow \cong \\
H^{2 k n-*}\left(T Y_{k}^{n}\right) & \stackrel{f}{\rightarrow} & H^{2(k+1) n-*}\left(T Y_{k+1}^{n}\right) .
\end{array}
$$

Lemma 3.2. The map $f$ corresponds to cupping with $a_{1} \cup a_{2} \cup \cdots \cup a_{n}$.

Proof. Let $V$ be a tubular neighborhood of $\operatorname{Div}_{k}^{n}$ in $\operatorname{Div}_{k+1}^{n}$. We have that $\operatorname{Div}_{k}^{n}$ is a (complex) codimension $n$ submanifold of $\operatorname{Div}_{k+1}^{n}$ and we can identify $V$ with the normal disc bundle to $\operatorname{Div}_{k}^{n} \subset \operatorname{Div}_{k+1}^{n}$. Denote by $\eta$ the entire normal bundle and let $M(\eta)=V / \partial V$ be the corresponding Thom space.

Note that we can compactify $\operatorname{Div}_{j}^{n}$ by adding a boundary term $\partial \operatorname{Div}_{j}^{n}$ (corresponding to its complement in $\left.S P^{j}\left(M_{g}\right)^{n}\right)$. Poincaré duality and the Thom isomorphism interlock in the following diagram of isomorphisms:

$$
\begin{array}{ccc}
H^{*}\left(\operatorname{Div}_{k}^{n}, \partial \operatorname{Div}_{k}^{n}\right) & \stackrel{\cup U}{\longrightarrow} & H^{*+2 n}\left(M(\eta), M\left(\eta_{\mid \partial}\right)\right) \\
\downarrow \cong & & \downarrow \cong \\
H_{2 k n-*}\left(\operatorname{Div}_{k}^{n}\right) & \stackrel{\Xi}{ } & H_{2 k n-*}\left(\operatorname{Div}_{k}^{n}\right)
\end{array}
$$

where $U$ is the Thom class and $M\left(\eta_{\mid \partial}\right)$ is the Thom space of the normal bundle $\eta$ restricted to $\partial \operatorname{Div}_{k}^{n}$. Note also that there is an (excision) isomorphism

$$
H^{*}(M(\eta))=H^{*}(V, \partial V) \stackrel{\cong}{\rightrightarrows} H^{*}\left(\operatorname{Div}_{k+1}^{n}, \operatorname{Div}_{k+1}^{n}-\operatorname{Div}_{k}^{n}\right)
$$

which then yields a map

$$
H^{*}\left(M(\eta), M\left(\eta_{\mid \partial}\right)\right) \rightarrow H^{*}\left(\operatorname{Div}_{k+1}^{n}, \partial \operatorname{Div}_{k+1}^{n}\right) .
$$

This is now enough to give a description of the map $f$, for we have that the Thom isomorphism (given by the top map in 3.3) combines with 3.4 to yield

$$
f: H^{*}\left(\operatorname{Div}_{k}^{n}, \partial \operatorname{Div}_{k}^{n}\right) \cong H^{*+2 n}\left(M(\eta), M\left(\eta_{\mid \partial}\right)\right) \rightarrow H^{*+2 n}\left(\operatorname{Div}_{k+1}^{n}, \partial \operatorname{Div}_{k+1}^{n}\right) .
$$


Write the tubular neighborhood $V$ of $\operatorname{Div}_{k}^{n}$ as

$$
V=\operatorname{Div}_{k}^{n} \times V_{x_{1}^{k}} \times \cdots \times V_{x_{n}^{k}}
$$

where $V_{x_{i}^{k}}$ is a small disc around $x_{i}^{k}$. The Thom class is by definition the orientation class $U \in H^{2 n}\left(V_{x_{1}^{k}} \times \cdots \times V_{x_{n}^{k}}, \partial\left(V_{x_{1}^{k}} \times \cdots \times V_{x_{n}^{k}}\right)\right)$. Since $H^{2}\left(V_{x_{i}^{k}}, \partial V_{x_{i}^{k}}\right)$ is generated by $a_{i}$, it is now clear that

$$
\begin{aligned}
U=a_{1} \ldots a_{n} \in H^{2}\left(V_{x_{1}^{k}}, \partial V_{x_{1}^{k}}\right) \otimes & \cdots \otimes H^{2}\left(V_{x_{n}^{k}}, \partial V_{x_{n}^{k}}\right) \\
& \hookrightarrow H^{2 n}\left(V_{x_{1}^{k}} \times \cdots \times V_{x_{n}^{k}}, \partial\left(V_{x_{n}^{k}} \times \cdots \times V_{x_{n}^{k}}\right)\right)
\end{aligned}
$$

and the proof is complete.

Corollary 3.5 (Segal). The “collar" inclusions $H_{*}\left(\operatorname{Div}_{k}^{n}\right) \stackrel{e_{*}}{\rightarrow} H_{*}\left(\operatorname{Div}_{k+1}^{n}\right)$ are injections.

\section{The Homology of Divisor Spaces}

In this section, we prove our main result ( 0.4 in the introduction and 4.14 below). So we start with the model $(\S 2)$

$$
D Y^{n}\left(M_{g}\right)=\left(S P^{\infty}\left(M_{g}, *\right)\right)^{n} \times_{t} S P^{\infty}\left(c M_{g}, *\right),
$$

where $t$ is the diagonal twisting described in 2.5. For simplicity, we will write $M$ for $M_{g}$ and $S P^{\infty}(M)$ for $S P^{\infty}\left(M_{g}, *\right)$.

We fix a diagonal approximation

$$
\Delta_{*}: C_{*}\left(S P^{\infty}(M)\right) \rightarrow C_{*}\left(S P^{\infty}(M)\right)^{\otimes^{n}} .
$$

This induces an action of $C_{*}\left(S P^{\infty}(M)\right)$ on $C_{*}\left(S P^{\infty}(M)\right)^{\otimes^{n}}$. On the other hand, the inclusion

$$
M \hookrightarrow c M, x \mapsto(0, x)
$$

induces an action of $C_{*}\left(S P^{\infty}(M)\right)$ on $C_{*}\left(S P^{\infty}(c M)\right)$. Using these actions, a chain complex for $D Y$ is given by

$$
C_{*}\left(S P^{\infty}(M)\right)^{\otimes n} \otimes_{C_{*}\left(S P^{\infty}(M)\right)} C_{*}\left(S P^{\infty}(c M)\right)
$$

and by Lemma 2.8, any other choice of $\Delta_{*}$ yields chain homotopic complexes.

At this point we need to describe the module structure of $C_{*}\left(S P^{\infty}(c M)\right)$ over $C_{*}\left(S P^{\infty}(M)\right)$, and for that purpose we need to review some constructions.

4.1. Milgram's Bar Construction. Infinite symmetric products provide models for topological bar constructions and classifying spaces, as was observed by Milgram [M1]-[M2].

Let $X$ be an associative topological monoid $\mu: X \times X \rightarrow X$ with $\mu$ cellular. We assume that $\mu$ has a unit ${ }^{*}$. Let $\sigma^{n}$ be the $n$-simplex which we parametrize as follows:

$$
\sigma^{n}=\left\{\left(t_{1}, t_{2}, \cdots, t_{n}\right) \mid 0 \leq t_{1} \leq \cdots \leq t_{n} \leq 1\right\} .
$$

The (acyclic) Milgram's bar construction on $X$ is the space

$$
E_{T}(X)=\coprod_{i=1}^{\infty} X \times \sigma^{i} \times X^{i} / \sim
$$


with identifications $\sim$ given as follows:

(i) $\quad\left(x_{0}, t_{1}, \cdots, t_{n}, x_{1}, \cdots, x_{n}\right)$ $\sim\left(x_{0}, t_{1}, \cdots, \hat{t}_{j}, \cdots, t_{n}, x_{1}, \cdots, \hat{x}_{j}, x_{j} x_{j+1}, \cdots, x_{n}\right)$ if $t_{j}=t_{j+1}$,

(ii) $\left(x_{0}, t_{1}, \cdots, t_{n}, x_{1}, \cdots, x_{n}\right) \sim\left(x_{0} x_{1}, t_{2}, \cdots, t_{n}, x_{2}, \cdots, x_{n}\right)$ if $t_{1}=0$,

(iii) $\left(x_{0}, t_{1}, \cdots, t_{n}, x_{1}, \cdots, x_{n}\right) \sim\left(x_{0}, t_{1}, \cdots, t_{n-1}, x_{1}, \cdots, x_{n-1}\right)$ if $t_{n}=1$,

(iv) $\left(x_{0}, t_{1}, \cdots, t_{n}, x_{1}, \cdots, x_{n}\right)$ $\sim\left(x_{0}, t_{1}, \cdots, \hat{t}_{j}, \cdots, t_{n}, x_{1}, \cdots, \hat{x}_{j}, \cdots, x_{n}\right)$ if $x_{j}=*$.

Clearly, $X$ acts freely on $E_{T}(X)$ by multiplying on the left and it turns out that $E_{T}(X)$ is contractible [M1]. This implies that the quotient space $B_{T}(X)=$ $E_{T}(X) / X$ is a classifying space for $X$. The space $B_{T}(X)$ is also referred to as the topological bar construction on $X$ (and we will sometimes write $B_{X}$ for $B_{T}(X)$.)

Let $E(A)$ and $B(A)$ denote respectively the acyclic and the reduced algebraic bar constructions on $A$. In our case, $A$ will be a differential graded, or $D G$, algebra. Suppose now that $X$ is an abelian $H$-space; then $C_{*}(X)$, the chain complex for $X$, is a DG algebra. There is a correspondence $\lambda: C_{*}\left(B_{T}(X)\right) \rightarrow B C_{*}(X)$ given on generators by

$$
\lambda\left(\sigma^{n} \times e_{1} \times \cdots \times e_{n}\right)=\left|e_{1}\right| \cdots\left|e_{n}\right| .
$$

Both $C_{*}\left(B_{T}(X)\right)$ and $B C_{*}(X)$ are bigraded and it can be checked that $\lambda$ is a differential bigraded algebra homomorphism. Actually, more is true:

Theorem 4.2 (Milgram). There is an isomorphism of differential bigraded algebras (dba);

$$
C_{*}\left(B_{T}(X)\right) \cong B\left(C_{*}(X)\right), C_{*}\left(E_{T}(X)\right) \cong E\left(C_{*}(X)\right) .
$$

Remark 4.3. We mentioned earlier that there is an interesting connection between infinite symmetric products and the classifying space construction above. Indeed, one can order points

$$
\left\langle\left(t_{1}, z_{1}\right), \ldots,\left(t_{n}, z_{n}\right)\right\rangle \in S P^{n}(\Sigma X)
$$

according to the ascending order of the $t_{i}$ 's. However there is an ambiguity whenever $t_{i}=t_{j}$, in which case we identify $\left\langle\left(t_{i}, z_{i}\right),\left(t_{i}, z_{j}\right)\right\rangle$ with $\left\langle t_{i},\left\langle z_{i} z_{j}\right\rangle\right\rangle$, where $\left\langle z_{i} z_{j}\right\rangle$ is the product in $S P^{\infty}(X)$. Of course when $t_{i}=0$ or $t_{i}=1$ we get the basepoint identification (in the suspension). It then follows that when elements of $S P^{\infty}(\Sigma X)$ are represented in the normal form $\left(t_{1} \leq t_{2} \leq \cdots \leq t_{n}, x_{1}, \ldots, x_{n}\right)$, the following homeomorphism becomes apparent:

$$
S P^{\infty}(\Sigma X)=B_{T}\left(S P^{\infty}(X)\right) .
$$

Corollary 4.4 (Milgram). $C_{*}\left(S P^{\infty}(\Sigma X, *)\right) \cong_{d b a} B\left(C_{*}\left(S P^{\infty}(X, *)\right)\right)$.

A similar statement holds for $c X$; that is, $S P^{\infty}(c X) \cong E_{T}\left(S P^{\infty}(X)\right)$ and there is a dba (i.e. differential bigraded algebra) isomorphism

$$
C_{*}\left(S P^{\infty}(c X, *)\right) \cong_{d b a} E\left(C_{*}\left(S P^{\infty}(X, *)\right)\right) .
$$

It then follows that

$$
C_{*}\left(S P^{\infty}(c M)\right) \cong E C_{*}\left(S P^{\infty}(M)\right)
$$

as modules over $C_{*}\left(S P^{\infty}(M)\right)$. Combining this with 4.1 gives

Lemma 4.5. $H_{*}\left(D Y^{n}(M) ; \mathbb{A}\right) \cong T o r^{C_{*}\left(S P^{\infty}(M, *)\right)}\left(\mathbb{A}, C_{*}\left(S P^{\infty}(M, *)\right)^{\otimes n}\right)$. 
4.2. The Collapse. The total space for $\operatorname{Tor}_{*} C_{*}\left(S P^{\infty}(M)\right)\left(\mathbb{A}, C_{*}\left(S P^{\infty}(M)\right)^{\otimes n}\right)$ is given by

$$
C_{*}\left(S P^{\infty}(M)\right)^{\otimes n} \otimes_{C_{*}\left(S P^{\infty}(M)\right)} E\left(C_{*}\left(S P^{\infty}(M)\right)\right) .
$$

We will write $\otimes_{\Delta_{*}}$ instead of $\otimes_{C_{*}(S P \infty(M))}$ for shorthand. Of course, filtering 4.6 by the number of bar degrees yields the classical homology Eilenberg-Moore spectral sequence.

Proposition 4.7. There is an embedding

$$
e: H_{*}\left(S P^{\infty}\left(M_{g}\right) ; \mathbb{A}\right) \hookrightarrow C_{*}\left(S P^{\infty}\left(M_{g}\right), \mathbb{A}\right)
$$

inducing an isomorphism

$$
\operatorname{Tor}^{H_{*}\left(S P^{\infty}\left(M_{g}\right)\right)}\left(\mathbb{A}, H_{*}\left(S P^{\infty}\left(M_{g}\right)\right)^{\otimes n}\right) \cong \operatorname{Tor}^{C_{*}\left(S P^{\infty}\left(M_{g}\right)\right)}\left(\mathbb{A}, C_{*}\left(S P^{\infty}\left(M_{g}\right)\right)^{\otimes n}\right) .
$$

From the Cartan-Moore comparison theorem ([McCl], corollary 7.6.), 4.7 would follow if $e$ is compatible with the module structures; that is if $e$ commutes with the diagonal action

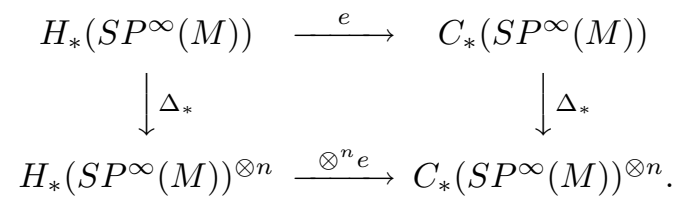

So first we record the existence of $e$ as a separate lemma.

Lemma 4.8. A chain complex for $S P^{\infty}\left(M_{g}\right)$ can be chosen so that there is an embedding e : $H_{*}\left(S P^{\infty}\left(M_{g}\right) ; \mathbb{Z}\right) \hookrightarrow C_{*}\left(S P^{\infty}\left(M_{g}\right), \mathbb{Z}\right)$.

Proof. We go back to the standard representation of $M_{g}$ as $M_{g} \simeq\left(\bigvee^{2 g} S^{1}\right) \cup_{[-]} D^{2}$, where $D^{2}$ is the top 2-cell attached via the commutator map [-] to a bouquet of $2 g$ one dimensional $S^{1}$ 's. The $i$-th copy of $S^{1}$ in $\bigvee^{2 g} S^{1}$ represents a one dimensional cell $e_{i}$ attached trivially to basepoint. If $*$ denotes the product in $S P^{\infty}(M)$, then we see dirctly that $e_{i_{1}} * e_{i_{2}} * \ldots * e_{i_{n}}, i_{j} \neq i_{k}$, and $e_{i} * D^{2}$ give genuine cells in $S P^{\infty}(M)$ (which can be thought of as the cross product cells). We also know that $S P^{n}\left(D^{2}\right), n \geq 1$, are cells of dimension $2 n$ (Lemma 1.5). We can consider then the complex $\mathcal{C}$ generated by the different products $e_{i_{1}} * e_{i_{2}} * \ldots * e_{i_{n}} * S P^{l}\left(D^{2}\right)$ with $i_{j} \neq i_{k}$. Since $\partial e_{i}=0, \partial D^{2}=0$, we notice that $\partial\left(e_{i} * S P^{n}\left(D^{2}\right)\right)=0$ and hence elements of $\mathcal{C}$ represent homology classes. As such, it is clear that $n !\left(S P^{n}\left(D^{2}\right)\right)=$ $[M]$, for this is simply equivalent to the statement that the projection map $M^{n} \rightarrow$ $S P^{n}(M)$ has degree $n$ !. It then follows that the $S P^{l}\left(D^{2}\right)$ 's generate a divided power algebra in $\mathcal{C}$. From Lemma 1.8 we see that $\mathcal{C} \cong H_{*}\left(S P^{\infty}(M)\right)$, and the embedding of the homology into the chain complex is constructed.

Proof of 4.7. The diagonal approximation $\Delta: M \rightarrow M^{n}$ can be extended multiplicatively (on each component) to a map $\Delta^{\infty}: S P^{\infty}(M) \rightarrow\left(S P^{\infty}(M)\right)^{n}$, and clearly $\Delta^{\infty}$ is homotopic to the diagonal on $S P^{\infty}(M)$. We have the following commuting diagram:

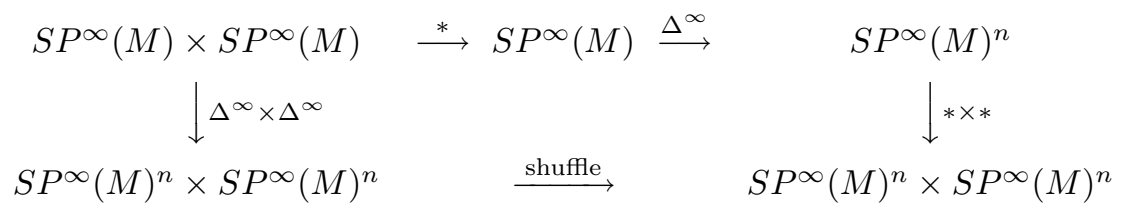


where as before shuff $\left(\left(x_{1}, \ldots, x_{n}\right),\left(y_{1}, \ldots y_{n}\right)\right)=\left(\left(x_{1}, y_{1}\right), \ldots,\left(x_{n}, y_{n}\right)\right)$. Note that shuff $_{*}$ is cellular. Now $\Delta^{\infty}$ is already cellular on $M$ by construction, and the diagram above shows that $\Delta_{*}^{\infty}$ must be cellular on $\mathcal{C}$. By standard considerations, $\Delta^{\infty}$ extends to a cellular map on all of $S P^{\infty}(M)$. The embedding $e: \mathcal{C} \hookrightarrow C_{*}\left(S P^{\infty}(M)\right)$ does commute with the diagonal action, and since $\mathcal{C}=H_{*}\left(S P^{\infty}\left(M_{g}\right)\right.$ the proposition follows by Cartan-Moore.

4.3. The Main Result. Write $\mathcal{A}=H_{*}\left(S P^{\infty}(M)\right)$. We now put a multigrading on $\operatorname{Tor}^{\mathcal{A}}\left(\mathbb{A}, \mathcal{A}^{\otimes n}\right)$ and derive Theorem 0.4. First, by Steenrod's splitting 1.6, the total space for $\operatorname{Tor}^{\mathcal{A}}\left(\mathbb{A}, \mathcal{A}^{n}\right)$ takes the form

$$
T o t=\bigoplus H_{*}\left(T_{k_{1}, \ldots, k_{n}} ; \mathbb{A}\right) \otimes_{\mathcal{A}} E(\mathcal{A}),
$$

where $T_{k_{1}, k_{2}, \ldots, k_{n}}=S P^{k_{1}}(M) \times \cdots \times S P^{k_{n}}(M) / V$ with

$$
V=\bigcup_{1 \leq i \leq n} S P^{k_{1}}(M) \times \cdots S P^{k_{i}-1} \times \cdots \times S P^{k_{n}}(M) .
$$

Since the algebra $\mathcal{A}$ is bigraded, so is $E(\mathcal{A})$. More precisely, let $a_{i} \in \mathcal{A}$ have bidegree $\left(\operatorname{deg}\left(a_{i}\right), \operatorname{fil}\left(a_{i}\right)\right)$ as in 1.7 ; then

$$
\text { bidegree }\left(a_{0}\left|a_{1}\right| a_{2}|\ldots| a_{n} \mid\right)=\left(n+\sum_{i=0}^{n} \operatorname{deg}\left(a_{i}\right), \sum \operatorname{fil}\left(a_{i}\right)\right),
$$

which means that the homological degree of $a_{0}\left|a_{1}\right| a_{2}|\ldots| a_{n} \mid \in E(\mathcal{A})$ is given by $\sum \operatorname{deg}\left(a_{i}\right)+n$, while the filtration degree is simply $\sum \operatorname{fil}\left(a_{i}\right)$ (this is not the bar degree). Define $E_{m, k}(\mathcal{A})$ to consist of all elements of bidegree $(m, k) \in E(\mathcal{A})$.

The boundary $\partial$ in $T o t$ is described as follows:

$$
\partial\left(\left|a_{1}\right| a_{2}|\ldots| a_{n} \mid\right)=\Delta_{*}\left(a_{1}\right) \otimes\left|a_{2}\right| \ldots\left|a_{n}\right|+\partial_{B}\left(\left|a_{1}\right| \ldots\left|a_{n}\right|\right),
$$

where $\partial_{B}$ is the reduced bar differential which in this case is given by

$$
\partial_{B}\left(\left|a_{1}\right| \ldots\left|a_{n}\right|\right)=(-1)^{i} \sum_{1}^{n-1}\left|a_{1}\right| \ldots\left|a_{i-1}\right| a_{i} a_{i+1}\left|a_{i+2}\right| \ldots\left|a_{n}\right| .
$$

By definition we have that $H_{*}(T o t, \partial)=\operatorname{Tor}^{\mathcal{A}}\left(\mathbb{A}, \mathcal{A}^{n}\right)$ for untwisted coefficients $\mathbb{A}$. Note that $\Delta_{*}: H_{*}\left(S P^{r}(M)\right) \rightarrow H_{*}\left(S P^{r}(M)\right)^{\otimes n}$ preserves the filtration degree $r$ and hence the total space $T o t$ splits as a sum of subchain complexes:

$$
\bigoplus_{l_{i} \leq k_{i}} \operatorname{Tot}_{l_{1}, \ldots, l_{n}}=\bigoplus_{l_{i}=r_{i}+j \leq k_{i}} H_{*}\left(T_{r_{1}, \ldots, r_{n}} ; \mathbb{A}\right) \otimes_{\mathcal{A}} E_{*, j}(\mathcal{A})
$$

The homology $H_{*}\left(\bigoplus_{l_{i} \leq k_{i}} \operatorname{Tot}_{k_{1}, \ldots, k_{n}}\right)$ coincides with $H_{*}\left(D Y_{k_{1}, \ldots, k_{n}}, \mathbb{A}\right)$, and this is a direct summand of $H_{*}(D Y, \mathbb{A})$. On the other hand, and by construction, $H_{*}\left(T Y_{k_{1}, \ldots, k_{n}}\right)$ is a quotient of $H_{*}\left(D Y_{k_{1}, \ldots, k_{n}}\right)$, which only sees elements of the exact filtration $\left(k_{1}, \ldots, k_{n}\right)$. When passing to this quotient, the diagonal term $\Delta_{*}$ gets reduced according to

$$
\Delta_{*}^{\mathrm{red}}: H_{*}\left(S P^{r}(M)\right) \stackrel{\Delta_{*}}{\longrightarrow}\left(H_{*}\left(S P^{r}(M)\right)\right)^{\otimes n} \stackrel{q}{\longrightarrow} H_{*}\left(S P^{r}(M), S P^{r-1}(M)\right)^{\otimes n} .
$$

We write

$$
{ }^{2} \operatorname{Tor}^{H_{*}\left(S P^{\infty}(M)\right)}\left(\mathbb{A}, H_{*}\left(S P^{\infty}(M)\right)^{\otimes n}\right)
$$


for the new tor term, where the action is understood to be reduced. This new action induces a new boundary ${ }^{2} \partial$ as in 4.10 , and in this case

$$
{ }^{2} \operatorname{Tot}_{k_{1}, \ldots, k_{n}}=\bigoplus_{r_{i}+j=k_{i}} H_{*}\left(T_{r_{1}, \ldots, r_{n}} ; \mathbb{A}\right) \otimes_{\Delta_{*}} E_{*, j}(\mathcal{A})
$$

is a subcomplex of $\left(T o t,{ }^{2} \partial\right)$. One then has

$$
H_{*}\left(T Y_{k_{1}, \ldots, k_{n}} ; \mathbb{A}\right) \cong 2 \operatorname{Tor}_{*, k_{1}, \ldots, k_{n}}^{H_{*}\left(S P^{\infty}(M)\right)}\left(\mathbb{A}, H_{*}\left(S P^{\infty}(M)\right)^{\otimes n}\right)=H_{*}\left({ }^{2} \operatorname{Tot}_{k_{1}, \ldots, k_{n}}\right),
$$

and these represent the homology classes of the exact filtration $\left(k_{1}, \ldots, k_{n}\right)$. When $k_{i}=k$, we shorten the notation $(* ; k, \ldots, k)$ to a bidegree notation $(* ; k)$ for simplicity. From now on, we drop the superscript ${ }^{2}$ Tor and write Tor with the understanding that the module action of $H_{*}\left(S P^{\infty}(M)\right)$ on the tensor product is reduced (cf. 4.11). The preceding discussion then yields

Theorem 4.14. For field coefficients $\mathbb{F}$, we have the following isomorphism:

$$
H_{*}\left(\operatorname{Div}_{k, \ldots, k}\left(M_{g}-*\right) ; \mathbb{F}\right) \cong \operatorname{Tor}_{2 n k-* ; k}^{H_{*}\left(S P^{\infty}\left(M_{g}\right)\right)}\left(\mathbb{F}, H_{*}\left(S P^{\infty}(M)\right)^{\otimes n}\right)
$$

with the module structure induced from $\Delta_{*}^{\text {red }}: H_{*}\left(S P^{\infty}(M)\right) \rightarrow H_{*}\left(S P^{\infty}(M)^{n}\right)$ in 4.11.

Proof. Apply the duality $H_{*}\left(T Y_{k, \ldots, k} ; \mathbb{F}\right) \cong H^{2 n k-*}\left(\operatorname{Div}_{k}^{n}(M-*) ; \mathbb{F}\right)$.

Remark 4.15. Note that $H_{*}\left(\operatorname{Div}_{k, \ldots, k}\left(M_{g}-*\right) ; \mathbb{F}\right)$ must vanish beyond the middle dimension $*>n k$, this being a pecularity of Stein spaces.

4.4. An Alternate Description. One could have filtered the space

$$
T Y^{n}=\bigcup D Y_{k_{1}, \ldots, k_{n}} / \cup_{j} D Y_{k_{1}, \ldots, k_{j}-1, \ldots, k_{n}}
$$

not by the number of bars but as follows. Write

$$
T Y^{n}=\bigcup T Y_{k_{1}, \ldots, k_{n}}=\bigcup_{r_{i}+l=k_{i}} T_{r_{1}, \ldots, r_{n}} \times_{t}\left(S P^{l}\left(c M_{g}\right) / S P^{l-1}\left(c M_{g}\right)\right)
$$

with filtration pieces

$$
\mathcal{F}^{j}=\bigcup_{\substack{l \leq j \\ r_{i}+l=k_{i}}}\left(T_{r_{1}, \ldots, r_{n}}\right) \otimes_{t}\left(S P^{l}\left(c M_{g}\right) / S P^{l-1}\left(c M_{g}\right)\right) .
$$

The same arguments as in $\S 4.3$ can now be expressed in the following form

Proposition 4.16. There exists a spectral sequence converging to $H_{*}\left(T Y_{k_{1}, \ldots, k_{n}}\right)$ with $E^{1}$ term

$$
E^{1}=\coprod_{r_{i}+j=k_{i}} H_{*}\left(T_{r_{1}, \ldots, r_{n}} ; \mathbb{F}\right) \otimes H_{*}\left(S P^{j}\left(\Sigma M_{g}\right), S P^{j-1}\left(\Sigma M_{g}\right), \mathbb{F}\right) .
$$

The spectral sequence collapses at $E^{1}$ for $n>2$.

Proof. It can easily be checked in light of $\S 4.3$ that

$$
d_{1}\left(c_{*} \otimes\left|a_{1}\right| \ldots\left|a_{l}\right|\right)=c_{*} \Delta_{*}^{\text {red }}\left(a_{1}\right) \otimes\left|a_{2}\right| \ldots\left|a_{l}\right|
$$

where $a_{i} \in H_{*}\left(S P^{\infty} M\right)$ and $\left|a_{1}\right| \ldots\left|a_{l}\right| \in H_{*}\left(S P^{\infty}(\Sigma M)\right)=H_{*}\left(B_{T}\left(S P^{\infty}(M)\right)\right)$ $=B\left(H_{*}\left(S P^{\infty} M\right)\right)$. This yields the first part of the proposition (cf. 4.12). That the spectral sequence collapses when $n>2$ is a corollary of the fact that $\Delta_{*}^{\text {red }}$ vanishes in this case (see Lemma 6.2). 
Remark 4.17. When $n=2$ we have $d_{1}(|M|) \neq 0$, and there are higher differentials $d_{p^{i}}$ described in Remark 6.16.

\section{The Rat Spaces}

When $g=0$, we have the homeomorphism described in the introduction:

$$
\operatorname{Div}_{k}^{n+1}\left(S^{2}-*\right)=\operatorname{Hol}_{k}^{*}\left(S^{2}, \mathbb{P}^{n}\right)=\operatorname{Rat}_{k}\left(\mathbb{P}^{n}\right) .
$$

Applying Theorem 4.14, we see that the module structure of $H_{*}\left(S P^{\infty}\left(S^{2}\right)\right)=\Gamma(a)$ on $H_{*}\left(S P^{\infty}\left(S^{2}\right)\right)^{n}$ is trivial, for in this case $\Delta_{*}\left(a=\left[S^{2}\right]\right)=a \otimes 1+1 \otimes a$ and hence by $4.11, \Delta_{*}^{\text {red }}(a)=0$. It follows that

$$
\operatorname{Tor}^{H_{*}\left(S P^{\infty}\left(S^{2}\right)\right)}\left(\mathbb{F}, H_{*}\left(S P^{\infty}\left(S^{2}\right)\right)^{n+1}\right)=\Gamma\left(a_{1}, \ldots, a_{n+1}\right) \otimes \operatorname{Tor}^{\Gamma(a)}(\mathbb{F}, \mathbb{F}) .
$$

We observe that we have an identification

$$
\operatorname{Tor}^{\Gamma(a)}(\mathbb{F}, \mathbb{F})=H_{*}\left(S P^{\infty}\left(S^{3}\right), \mathbb{F}\right)=\coprod_{i} H_{*}\left(S P^{i}\left(S^{3}\right), S P^{i-1}\left(S^{3}\right), \mathbb{F}\right)
$$

and can then write

$$
H_{*}\left(T Y_{k}^{n+1}\left(S^{2}\right) ; \mathbb{F}\right) \cong \coprod_{j} \gamma_{j}\left(a_{1} \ldots a_{n+1}\right) H_{*-2(n+1) j}\left(S P^{k-j}\left(S^{3}\right), S P^{k-j-1}\left(S^{3}\right) ; \mathbb{F}\right) .
$$

We can consider the inclusion $\operatorname{Rat}\left(\mathbb{P}^{1}\right) \hookrightarrow \Omega^{2} S^{2}$. The space $\Omega_{0}^{2} S^{2} \simeq \Omega^{2} S^{3}$ stably splits (Snaith) as an infinite bouquet

$$
\Omega^{2} \Sigma^{2} S^{1} \simeq_{s} \bigvee_{0}^{\infty} D_{k}
$$

where $D_{k}=F(\mathbb{C}, k) \wedge \mathcal{S}_{k} S^{(k)}$ are the building blocks of the May-Milgram model for $S^{1}$ (here $S^{(k)}$ denotes the $k$-fold smash of $S^{1}$ with itself). It is known [BCM] that there is a duality isomorphism

$$
H_{*}\left(D_{k}, \mathbb{F}\right) \cong H^{4 k-*}\left(S P^{k}\left(S^{3}\right), S P^{k-1}\left(S^{3}\right), \mathbb{F}\right) .
$$

The identity 5.1 and the duality $H_{2 k(n+1)-*}\left(T Y_{k}^{n+1}, \mathbb{F}\right) \cong \tilde{H}^{*}\left(\operatorname{Rat}_{k}\left(\mathbb{P}^{n}\right), \mathbb{F}\right)$ combine to yield

$$
\begin{aligned}
H_{*}\left(\operatorname{Rat}_{k}\left(\mathbb{P}^{n}\right), \mathbb{F}\right) & =H^{2 k(n+1)-*}\left(T Y_{k}^{n+1}, \mathbb{F}\right) \\
& =\bigoplus_{j} H^{2(n+1)(k-j)-*}\left(S P^{k-j}\left(S^{3}\right), S P^{k-j-1}\left(S^{3}\right) ; \mathbb{F}\right) \\
& \left.=\bigoplus_{4(k-j)-2(n+1)(k-j)+*} H_{k-j} ; \mathbb{F}\right) \\
& =\bigoplus_{j} H_{*-(2 n-2)(k-j)}\left(D_{k-j} ; \mathbb{F}\right) .
\end{aligned}
$$

Proposition $5.2([\mathrm{C} 2 \mathrm{M} 2])$. $H_{*}\left(\operatorname{Rat}_{k}\left(\mathbb{P}^{n}\right), \mathbb{F}\right) \cong H_{*}\left(\bigvee_{j=1}^{k} \Sigma^{(2 n-2) j} D_{j}, \mathbb{F}\right)$.

Corollary 5.3 (Segal). Let $\operatorname{Rat}_{\infty}\left(\mathbb{P}^{n}\right)$ be the direct limit induced from the system of collar inclusions $\operatorname{Rat}_{k}\left(\mathbb{P}^{n}\right) \rightarrow \operatorname{Rat}_{k+1}\left(\mathbb{P}^{n}\right)$. Then

$$
H_{*}\left(\operatorname{Rat}_{\infty}\left(\mathbb{P}^{n}\right), \mathbb{Z}\right) \cong H_{*}\left(\Omega_{0}^{2} S^{2 n+1}, \mathbb{Z}\right) .
$$


Remark 5.4. Cohen and Shimamoto show that the isomorphism in 5.2 is induced from an actual homotopy equivalence $\operatorname{Rat}_{k}\left(\mathbb{P}^{n}\right) \simeq C_{k}\left(\mathbb{C}, S^{2 n-1}\right)$ whenever $n>1$. We refer to [CS] for a definition of the labelled configuration space $C_{k}\left(\mathbb{C}, S^{2 n-1}\right)$ and its relation with $\Omega^{2} S^{2 n-1}$.

\section{The Positive Genus Case}

In this section we determine the full structure of $\operatorname{Tor}^{\mathcal{A}}\left(\mathbb{F}, \mathcal{A}^{\otimes n}\right)$ for $g \geq 1$, and for both rational and $\mathbb{Z}_{p}$ coefficients. We start by making explicit the action of $\mathcal{A}$ on $\mathcal{A}^{\otimes n}$. The algebra $\mathcal{A}=H_{*}\left(S P^{\infty}\left(M_{g}\right)\right)$ acts on $\mathcal{A}^{\otimes n}$ via the prescription

$$
x \cdot\left(c_{1} \otimes \cdots \otimes c_{n}\right)=\nu_{*}\left(\Delta_{*}^{\mathrm{red}}(x) \otimes\left(c_{1} \otimes \cdots \otimes c_{n}\right)\right),
$$

where $\nu$ is the componentwise symmetric product multiplication and where $\Delta_{*}^{\text {red }}$ is as in 4.11. Now recall that $\mathcal{A}$ has generators the 1-dimensional classes $e_{i}, 1 \leq i \leq 2 g$, which are primitive, as well as the top orientation class $[M]$.

Notation. We denote by $e_{i ; r}$ the element $1 \otimes \cdots \otimes e_{i} \otimes \cdots \otimes 1$, where $e_{i}$ is in the $r^{t h}$ position, $1 \leq r \leq n$. By $e_{i ; r} e_{j ; s}$ for $r<s$ we then mean $1 \otimes \cdots \otimes e_{i} \otimes \cdots \otimes e_{j} \otimes \cdots \otimes 1$.

Lemma 6.1. $\Delta_{*}\left(\left[M_{g}\right]\right)=\sum_{r}\left[M_{g}\right]_{r}+\sum_{j=1}^{g} \sum_{r<s}\left(e_{2 j ; r} e_{2 j-1 ; s}-e_{2 j-1 ; r} e_{2 j ; s}\right)$.

Proof. There is a natural collapse map from $M_{g}$ to a wedge of $g$ tori $T_{1} \vee \cdots \vee T_{g}$ inducing an isomorphism in $H_{1}$ and such that the image of $[M]$ is $\sum\left[T_{i}\right]$. We have that $T_{i}=S^{1} \times S^{1}$ and $H_{*}\left(T_{i}\right)=\Lambda\left(e_{2 i-1}, e_{2 i}\right)$. It is easy to see that

$$
\Delta_{*}\left[T_{i}\right]=\sum\left[T_{i}\right]_{r}+\sum_{r<s}\left(e_{2 i ; r} e_{2 i-1 ; s}-e_{2 i-1 ; r} e_{2 i ; s}\right)
$$

and hence by adding these up the lemma follows.

Lemma 6.2. $\Delta_{*}^{\mathrm{red}}\left(e_{i}\right)=0, \forall 1 \leq i \leq 2 g, n \geq 2$. On the other hand,

$$
\Delta_{*}^{\mathrm{red}}([M])= \begin{cases}\sum_{j=1}^{g}\left(e_{2 j} \otimes e_{2 j-1}-e_{2 j-1} \otimes e_{2 j}\right), & \text { if } n=2, \\ 0, & \text { if } n>2 .\end{cases}
$$

Proof. Note that $e_{2 j-1} \otimes e_{2 j}-e_{2 j} \otimes e_{2 j-1} \in H_{1}(M, *)^{\otimes 2} \subset H_{2}\left(S P^{\infty}(M)^{2}\right)$ (here $n=2)$ and this is non-trivial in the image of $\Delta_{*}^{\text {red }}$. The rest is a direct consequence of 4.11 .

Corollary 6.3. Suppose $n>2$. Then

$$
\begin{aligned}
\operatorname{Tor}^{\mathcal{A}}\left(\mathbb{F}, \mathcal{A}^{\otimes n}\right) & \cong \mathcal{A}^{\otimes n} \otimes \operatorname{Tor}^{\mathcal{A}}(\mathbb{F}, \mathbb{F}) \\
& \cong \mathcal{A}^{\otimes n} \otimes H_{*}\left(S P^{\infty}\left(\Sigma M_{g}, *\right) ; \mathbb{F}\right) \\
& \cong \mathcal{A}^{\otimes n} \otimes_{i} \operatorname{Tor}^{\Lambda\left(e_{i}\right)}(\mathbb{F}, \mathbb{F}) \otimes \operatorname{Tor}^{\Gamma([M])}(\mathbb{F}, \mathbb{F}) .
\end{aligned}
$$

Proof. Since both $\Delta_{*}^{\text {red }}\left(e_{i}\right)$ and $\Delta_{*}^{\text {red }}([M])$ vanish for $n>2$, it follows that $\Delta_{*}^{\text {red }}$ vanishes on the generators of $\mathcal{A}$ and hence induces a trivial action on $\mathcal{A}^{\otimes n}$ whenever $n>2$. This gives the first isomorphism. The last two identities are a consequence of the embedding $H_{*}\left(S P^{\infty}(M)\right) \hookrightarrow C_{*}\left(S P^{\infty}(X)\right)$ (4.7) and of Cartan-Moore; i.e.

$$
\begin{aligned}
H_{*}\left(S P^{\infty}(\Sigma X) ; \mathbb{F}\right) & =\operatorname{Tor}^{H_{*}\left(S P^{\infty}(M)\right)}(\mathbb{F}, \mathbb{F})=\operatorname{Tor}^{\Lambda\left(e_{1}, \cdots, e_{2 g}\right) \otimes \Gamma([M])}(\mathbb{F}, \mathbb{F}) \\
& =\bigotimes_{i} \operatorname{Tor}^{\Lambda\left(e_{i}\right)}(\mathbb{F}, \mathbb{F}) \otimes \operatorname{Tor}^{\Gamma([M])}(\mathbb{F}, \mathbb{F}) . \quad \square
\end{aligned}
$$

We now describe $H_{*}\left(S P^{n}(\Sigma M) ; \mathbb{F}\right)$ for $\mathbb{F}=\mathbb{Q}$ and $\mathbb{F}=\mathbb{Z}_{p}$. 
6.1. The Homology of $S P^{\infty}\left(\Sigma M_{g}\right)=B_{S P^{\infty}\left(M_{g}\right)}$. The acyclic bar construction for $\Lambda(e)$ over $\mathbb{Z}$ gives rise to a minimal resolution which is generated at each level $B_{i}(\Lambda(e)$ ) by elements of the form $|e| \cdots|e|$ or $e|e| \cdots|e|$ (\# of bars is $i$ ) and has boundary $\partial|e| e|\cdots| e|=e| e|\cdots| e \mid$. The generators $|e| \cdots|e|$ generate a divided power algebra (under the shuffle product), and it is readily seen that

$$
\operatorname{Tor}_{*, *}^{\Lambda(e)}(\mathbb{Z}, \mathbb{Z})=\Gamma(|e|) .
$$

The case of divided power algebras is harder. When $\mathbb{F}=\mathbb{Q}$, divided power algebras turn into polynomial algebras, and so in this case $\operatorname{Tor}^{\Gamma(a)}(\mathbb{Q}, \mathbb{Q})=\Lambda(|a|)$, implying that

$$
H_{*}\left(S P^{\infty}(\Sigma M) ; \mathbb{Q}\right)=\mathbb{Q}\left(\left|e_{1}\right|, \ldots,\left|e_{2 g}\right|\right) \otimes \Lambda([M]) .
$$

When $\mathbb{F}=\mathbb{Z}_{p}$, we see that $a^{p}=p ! \gamma_{p}=0$. Similarly, $\gamma_{p^{i}}^{p}$ is also zero. This shows that each $\gamma_{p^{i}}$ generates a truncated polynomial algebra

$$
P_{T}(a, p)=\mathbb{Z}_{p}\left[a, a^{2}, \cdots, a^{p-1}\right] / a^{p}=0 .
$$

Lemma 6.5 (Cartan). $\left\{\gamma_{p^{i}}, i \geq 0\right\}$ generate $\Gamma(a)$ as an algebra over $\mathbb{Z}_{p}$ and

$$
\Gamma(a) \otimes \mathbb{Z}_{p} \cong P_{T}(a, p) \otimes P_{T}\left(\gamma_{p}, p\right) \otimes \cdots \otimes P_{T}\left(\gamma_{p^{i}}, p\right) \otimes \cdots .
$$

We assume in what follows that $a$ has even degree (for our purpose $a=[M]$ ). One can construct a minimal resolution for $P_{T}(a, p)$ (over $\mathbb{Z}_{p}$ ) which is generated by elements

with boundary

$$
\left\{\left|a^{p-1}\right| a|\cdots| a^{p-1}|a|,|a|\left|a^{p-1}\right| a|\cdots| a^{p-1}|a|\right\}
$$

$$
\partial \underbrace{\left|a^{p-1}\right| a|\cdots| a^{p-1}|a|}_{i}=a^{p-1} \underbrace{\left|a^{p-1}\right| a|\cdots| a^{p-1}|a|}_{i-1} .
$$

As an algebra under the shuffle product, it is checked that

Lemma 6.6.

$$
\operatorname{Tor}^{P_{T}(a, p)}\left(\mathbb{Z}_{p}, \mathbb{Z}_{p}\right) \cong \Lambda(|a|) \otimes \Gamma\left(\left|a^{p-1}\right| a \mid\right), \quad p>2 .
$$

When $p=2$, then $P_{T}(a, 2)=\Lambda(a)$ and $\operatorname{Tor}^{P_{T}(a, 2)}\left(\mathbb{Z}_{2}, \mathbb{Z}_{2}\right) \cong \Gamma[|a|]$.

Remark 6.7. The element $|a| a^{p-1} \mid$ in the bar construction is known as the transpotence of $a$. It can be checked that $\beta\left(\left|\gamma_{p^{i+1}}\right|\right)=\left|\gamma_{p^{i}}^{p-1}\right| \gamma_{p^{i}} \mid$, where $\beta$ is the mod- $p$ Bockstein.

Remark 6.8. The generators $\left|e_{i}\right|,|M|$, all represent homology classes in $H_{*}(\Sigma M) \subset$ $H_{*}\left(S P^{\infty}(\Sigma M)\right)$ and this explains why they are referred to as suspension classes. All generators in $H_{*}\left(S P^{\infty}(\Sigma M)\right)$ are assigned a bidegree as in Remark 1.7 and we find that

$$
\begin{array}{cc}
\text { Generator } & \text { Bigrading } \\
\left|e_{i}\right| & (2 ; 1) \\
|M| & (3 ; 1) \\
\left|\gamma_{p^{i}}\right| & \left(2 p^{i}+1 ; p^{i}\right) .
\end{array}
$$

Bidegrees are additive; for example the bidegree of $\left|\gamma_{p^{i}}\right| \gamma_{p^{j}} \mid$ is $\left(2\left(p^{i}+p^{j}\right)+2 ; p^{i}+p^{j}\right)$. If we let $h_{2 p^{i}+1, p^{i}}=\left|\gamma_{p^{i}}\right|$, we can then write

$$
\operatorname{Tor}^{\Gamma(a)}\left(\mathbb{Z}_{p}, \mathbb{Z}_{p}\right) \cong \Lambda\left(|a|, \ldots, h_{2 p^{i}+1, p^{i}}, \ldots\right) \otimes \Gamma\left(\beta h_{2 p+2, p}, \ldots, \beta h_{2 p^{i}+2, p^{i}}, \ldots\right)
$$

and this describes the algebra $H_{*}\left(S P^{\infty}\left(S^{3}\right) ; \mathbb{Z}_{p}\right)=H_{*}\left(K(\mathbb{Z}, 3) ; \mathbb{Z}_{p}\right)$. Generally we have the following. 
Lemma 6.9. The homology $H_{*}\left(S P^{n}\left(\Sigma M_{g}\right) ; \mathbb{F}\right)$ is given by those classes of bidegree $(* ; i)$ with $i \leq n$, in

$$
\Gamma\left(\left|e_{1}\right|, \ldots,\left|e_{2 g}\right|\right) \otimes \operatorname{Tor}^{\Gamma(a)}(\mathbb{F}, \mathbb{F}) .
$$

6.2. Homology Calculations, $n>2$. The preceding discussion as well as Corollary 6.3 show that for $n>2$

$$
\begin{aligned}
\operatorname{Tor}^{\mathcal{A}}\left(\mathbb{F}, \mathcal{A}^{\otimes n}\right)= & \Gamma\left(a_{1}, \ldots, a_{n}\right) \otimes \Gamma\left(\left|e_{1}\right|, \ldots,\left|e_{2 g}\right|\right) \otimes \Lambda\left(e_{1}, \ldots, e_{2 n g}\right) \\
& \otimes \operatorname{Tor}^{\Gamma([M])}(\mathbb{F}, \mathbb{F}),
\end{aligned}
$$

the terms of $(n+1)$-grading $(*, k \ldots, k)$ making up all of $H_{2 n k-*}\left(\operatorname{Div}_{k}(M-* ; \mathbb{F})\right.$.

Proposition 6.11. Assume $n>2$ and $g \geq 1$ and consider the algebra

$$
\Lambda\left(f_{1 ; 1}, \ldots, f_{2 n g ; 1}, \ldots, f_{1 ; n}, \ldots, f_{2 g ; n}\right) \otimes \Lambda(E) \otimes \mathbb{Q}\left(h_{1}, \ldots, h_{2 g}\right) .
$$

This algebra is $(n+1)$-graded according to $f_{i ; r} \mapsto(1 ; 0, \ldots, 1, \ldots, 0)$ with 1 in the $r+1$ position, $1 \leq r \leq n, E \mapsto(2 n-3 ; 1, \ldots, 1)$ and $h_{j} \mapsto(2 n-2 ; 1, \ldots, 1)$. The multigrading is additive. The homology groups $H_{*}\left(\operatorname{Div}_{k}\left(M_{g}-*\right) ; \mathbb{Q}\right)$ are now given by those elements of multidegree $\left(* ; i_{1}, \ldots, i_{n}\right)$ with $i_{j} \leq k$.

Proof. With $\mathbb{Q}$ coefficients 6.10 takes the form

$$
\mathbb{Q}\left(a_{1}, \ldots, a_{n}\right) \otimes \mathbb{Q}\left(\left|e_{1}\right|, \ldots,\left|e_{2 g}\right|\right) \otimes \Lambda\left(e_{1}, \ldots, e_{2 n g}\right) \otimes \Lambda(|M|) .
$$

It is now a matter of counting the multidegree $(2 n k-* ; k, \ldots, k)$ elements. The degree one generators are represented by $a_{1}^{k} \ldots e_{i ; r} a_{j}^{k-1} \ldots a_{n}^{k}$ in $H_{2 n k-1}\left(T Y_{k}\right), 1 \leq$ $i \leq 2 g$ and $1 \leq r \leq n$, and to them correspond the $f_{i ; r} \in H_{1}\left(\operatorname{Div}_{k}(M-*) ; \mathbb{Q}\right)$. Similarly, $|M|$ is Poincaré dual to an element $E$ of the right filtration and of homology degree $2 n-3$, whereas the $h_{j}$ 's are dual to the $\left|e_{j}\right|$ 's. Here $\mathbb{Q}\left(a_{1}, \ldots, a_{n}\right)$ serves as a "calibrating" factor, and the calculation follows.

When $\mathbb{F}=\mathbb{Z}_{p}, p$ odd, we can facilitate the counting by dualizing $\operatorname{Tor}^{\mathcal{A}}\left(\mathbb{Z}_{p}, \mathcal{A}^{\otimes n}\right)$. Divided power algebras turn into polynomial algebras and we get the total space

$$
\begin{aligned}
\mathbb{Z}_{p}\left(a_{1}, \ldots, a_{n}\right) \otimes \Lambda\left(e_{1}, \ldots, e_{2 n g}\right) & \otimes \mathbb{Z}_{p}\left(\left|e_{1}\right|, \ldots,\left|e_{2 g}\right|\right) \\
\otimes \Lambda\left(|M|,\left|\gamma_{p}\right|, \ldots,\left|\gamma_{p^{i}}\right| \ldots\right) & \otimes \mathbb{Z}_{p}\left(\left|M^{p-1}\right| M|, \ldots,| \gamma_{p^{i}}^{p-1}\left|\gamma_{p^{i}}\right|, \ldots\right) .
\end{aligned}
$$

Here we ought to write $e_{i}^{*},\left|e_{i}\right|^{*},[M]^{*}$ for the classes above, but for simplicity we leave that out. Our calibration procedure leads generators $e_{i ; r}$ as well as

$$
\begin{array}{cccc} 
& h_{i} & E_{i} & H_{i} \\
(n+1) \text {-degree } & (2(n-1) ; 1, \ldots, 1) & \left(2(n-1) p^{i}-1 ; p^{i}, \ldots, p^{i}\right) & \left(2(n-1) p^{i} ; p^{i}, \ldots, p^{i}\right) .
\end{array}
$$

Lemma 6.12. Assume $n>2$ and $p$ odd; then $H_{*}\left(\operatorname{Div}_{k}^{n}\left(M_{g}-*\right) ; \mathbb{Z}_{p}\right)$ is given by those classes in

$$
\begin{aligned}
& \bigotimes_{1 \leq r \leq n} \Lambda\left(e_{1 ; r}, \ldots, e_{2 g ; r}\right) \otimes \mathbb{Z}_{p}\left(h_{1}, \ldots, h_{2 g}\right) \\
& \otimes \Lambda\left(E_{1}, \ldots, E_{j}, \ldots\right) \otimes \mathbb{Z}_{p}\left(H_{1}, \ldots, H_{j}, \ldots\right)
\end{aligned}
$$

of multidegree $\left(* ; i_{1}, \ldots, i_{n}\right)$ with $i_{j} \leq k$. 
6.3. Homology Calculations, $n=2$. As pointed out in 6.3, the action of $\mathcal{A}$ on $\mathcal{A}^{\otimes 2}$ is not trivial. The Tor term $\operatorname{Tor}^{\mathcal{A}}(\mathbb{F}, \mathcal{A} \otimes \mathcal{A})$ takes the form

$$
\Gamma\left(a_{1}, a_{2}\right) \otimes \Gamma\left(\left|e_{1}\right|, \ldots,\left|e_{2 g}\right|\right) \otimes \operatorname{Tor}^{\Gamma([M])}\left(\mathbb{F}, \Lambda\left(e_{1}, \ldots, e_{4 g}\right)\right),
$$

and the calculation boils down to understanding the term on the far right. The boundary here in the total space $\Lambda\left(e_{1}, \ldots, e_{4 g}\right) \otimes E(\Gamma[M])$ is generated by $\partial(|M|)=$ $\sum_{i=1}^{g} e_{2 i-1} e_{2 i}-e_{2 i} e_{2 i-1}$ (cf. Lemma 6.2). By reordering the $e_{i}$ 's and renaming, we can rewrite it as $\partial(|M|)=\sum_{i=1}^{2 g} e_{2 i-1} e_{2 i}$. Assume for now that $\mathbb{F}=\mathbb{Q}$. We can rewrite 6.13 as follows:

$$
H_{*}\left(\mathcal{W}_{g} ; \mathbb{Q}\right) \otimes \mathbb{Q}\left(h_{1}, \cdots, h_{2 g}\right) \otimes \mathbb{Q}\left(a_{1}, a_{2}\right),
$$

where $\mathcal{W}_{g}$ is the complex

$$
\Lambda\left(e_{1}, \cdots, e_{4 g}\right) \otimes \Lambda|M| \stackrel{\partial}{\rightarrow} \Lambda\left(e_{1}, \cdots, e_{4 g}\right), \partial(|M|)=e_{1} e_{2}+e_{3} e_{4}+\cdots+e_{4 g-1} e_{4 g} .
$$

By taking Poincaré duals we get

Lemma 6.14. Let $\overline{\mathcal{W}}_{g}$ denote the complex

$$
\Lambda\left(e_{1}, \ldots, e_{4 g}, f\right) \rightarrow \Lambda\left(e_{1}, \ldots, e_{4 g}\right), \delta f=e_{1} e_{2}+\cdots+e_{4 g-1} e_{4 g} .
$$

Then

$$
H_{*}\left(\operatorname{Div}_{k}^{2}\left(M_{g}-*\right) ; \mathbb{Q}\right) \subset H_{*}\left(\overline{\mathcal{W}}_{g}, \mathbb{Q}\right) \otimes \mathbb{Q}\left(h_{1}, \ldots, h_{2 g}\right)
$$

consists of elements with tridegrees $(* ; i, j), i, j \leq k$, where tridegrees are assigned as follows: $e_{\mathrm{odd}} \mapsto(1 ; 1,0), e_{\mathrm{even}} \mapsto(1 ; 0,1), f \mapsto(1 ; 1,1), h_{i} \mapsto(2 ; 1)$.

The complex $\overline{\mathcal{W}}_{g}$ has been studied in both $[\mathrm{BC}]$ and $[\mathrm{BCM}]$, and its Betti numbers have been completely determined. It is shown there for instance that the map

$$
\bigcup\left(e_{1} e_{2}+\cdots+e_{4 g-1} e_{4 g}\right): \Lambda\left(e_{1}, \cdots, e_{4 g}\right) \rightarrow \Lambda\left(e_{1}, \cdots, e_{4 g}\right)
$$

is injective in degrees $\leq 2 g$ and surjective in degrees $\geq 2 g$. Moreover, if $\nu(i, g)$ denotes the rank of $H_{i}\left(\overline{\mathcal{W}}_{g} ; \mathbb{Q}\right)$, then we have

Lemma 6.15 $([\mathrm{BCM}]) \cdot \nu(i, g)=0$ for $i>4 g+1$, and otherwise

$$
\nu(i, g)= \begin{cases}\left(\begin{array}{c}
4 g \\
i
\end{array}\right)-\left(\begin{array}{c}
4 g \\
i-2
\end{array}\right) & \text { for } i \leq 2 g \\
\left(\begin{array}{c}
4 g \\
i-1
\end{array}\right)-\left(\begin{array}{c}
4 g \\
i+1
\end{array}\right) & \text { for } 2 g<i \leq 4 g+1 .\end{cases}
$$

Remark 6.16. When $\mathbb{F}=\mathbb{Z}_{p}$, the boundary terms take the form

$$
\begin{aligned}
\partial\left(\left|\gamma_{p^{i}}\right|\right) & =\frac{1}{p^{i}}\left(\sum_{1}^{g} e_{2 i-1} e_{2 i}\right)^{p^{i}}, \\
\partial\left(\left|\gamma_{p^{i}}^{p-1}\right| \gamma_{p^{i}} \mid\right) & =\left[\frac{1}{p^{i}}\left(\sum_{1}^{g} e_{2 i-1} e_{2 i}\right)^{(p-1) p^{i}}\right]\left|\gamma_{p^{i}}\right| .
\end{aligned}
$$

These last differentials correspond to the Kudo differential in the Serre spectral sequence associated to the quasi-fibration $S P^{\infty}(M)^{n} \rightarrow D Y \rightarrow S P^{\infty}(\Sigma M)$. They also describe the $d_{p^{i}}$ in $\S 4.4$.

Example 6.17. As an example, we carry out the calculation for $T=M_{1}$, a genus 1 surface, $n=2$ and $p=2$. The complex at hand can be written as

$$
\Lambda\left(e_{1}, e_{2}, e_{3}, e_{4}\right) \otimes \mathbb{Z}_{2}\left(\left|e_{1}\right|,\left|e_{2}\right|,|T|,\left|\gamma_{2}\right|, \ldots,\left|\gamma_{2^{i}}\right| \ldots\right)
$$


on generators with tridegrees: $e_{1}, e_{3} \mapsto(1 ; 1,0), e_{2}, e_{4} \mapsto(1 ; 0,1),\left|e_{i}\right| \mapsto(2 ; 1,1)$, $|T| \mapsto(1 ; 1,1)$ and $\left|\gamma_{2^{i}}\right| \mapsto\left(2^{i+1}-1 ; 2^{i}, 2^{i}\right)$. The coboundary is given by

$$
\delta(|T|)=e_{1} e_{2}+e_{3} e_{4}, \delta\left(\left|\gamma_{2^{i}}\right|\right)=\frac{1}{2^{i}}\left(e_{1} e_{2}+e_{3} e_{4}\right)^{2^{i}} .
$$

This implies that $\delta\left(\left|\gamma_{2}\right|\right)=e_{1} e_{2} e_{3} e_{4}$ and $\delta\left(\left|\gamma_{2^{i}}\right|\right)=0$ for $i \geq 2$. We're interested in all elements of tridegree $(*, i, j), i, j \leq k$. For example, we have

Lemma 6.18. The Poincaré series for $H_{*}\left(\operatorname{Div}_{k}^{2}(T-*) ; \mathbb{F}_{2}\right), k=1,2$, are given by

$$
P(x)=1+4 x+5 x^{2}(k=1), P(x)=1+4 x+7 x^{2}+9 x^{3}+6 x^{4}(k=2) .
$$

\section{Homotopy Constructions}

We start with a fibration sequence due to Segal, which along with the scanning map, also first constructed in $[\mathrm{S}]$, constitutes the main tool in setting up the correspondence between divisor spaces and mapping spaces. We denote by $\mathbb{P}^{n}$ the $n$th complex projective space, and we use $\mathbb{P}$ and $\mathbb{P}^{\infty}$ interchangeably for the infinite complex space.

7.1. Fat-Wedge Fibrations. Let $W_{n} \mathbb{P}$ denote the $n^{\text {th }}$ fat wedge of $\mathbb{P}^{\infty}$; that is, $W_{n} \mathbb{P}$ is the subset of $\left(\mathbb{P}^{\infty}\right)^{n}$ consisting of all $n$-tuples with at least one entry equal to the basepoint in $\mathbb{P}^{\infty}$ (we sometimes write $W_{n}$ for $W_{n} \mathbb{P}$ ). Of course $W_{1}=\left\{x_{0}\right\}$ and $W_{2}=\mathbb{P}^{\infty} \vee \mathbb{P}^{\infty}$. One has the following

Lemma 7.1 (Segal). There is a fibration sequence

$$
\left(S^{1}\right)^{n} \rightarrow \mathbb{P}^{n} \rightarrow W_{n+1} \mathbb{P} \stackrel{\theta}{\rightarrow}\left(\mathbb{P}^{\infty}\right)^{n}=B\left(S^{1}\right)^{n} .
$$

The projection $\theta$ is described in $[\mathrm{S}], \S 2$. When $n=1$, this is the folding map (i.e. the restriction of $\left.\theta: \mathbb{P} \times \mathbb{P} \rightarrow \mathbb{P}, \theta(a, b)=a \cdot b^{-1}\right)$. In this particular case, the fiber of $\theta$ is the total space of the fibration over $\mathbb{P} \vee \mathbb{P}$ induced from the path/loop fibration $S^{1} \rightarrow S^{\infty} \rightarrow \mathbb{P}$ and this is seen to be $S^{\infty} \cup_{S^{1}} S^{\infty} \simeq \Sigma S^{1}=S^{2}$.

Lemma 7.2. There is a fibration

$$
S^{2 n+1} \rightarrow W_{n+1} \mathbb{P} \hookrightarrow\left(\mathbb{P}^{\infty}\right)^{n+1} .
$$

Proof. The proof proceeds by induction. In the case $n=1$, we have the inclusion $W_{2}=\mathbb{P} \vee \mathbb{P} \hookrightarrow \mathbb{P} \times \mathbb{P}$. A general result of Ganea states that the homotopy fiber of the inclusion $X \vee Y \hookrightarrow X \times Y$ is $\Omega(X) * \Omega(Y) \simeq \Sigma(\Omega(X) \wedge \Omega(Y))$ (here $*$ denotes the join product.) In our case, the fiber then becomes $\Sigma(\Omega \mathbb{P} \wedge \Omega \mathbb{P})) \simeq \Sigma\left(S^{1} \wedge S^{1}\right)=S^{3}$.

For $n>1$, the fiber of $W_{n+1} \hookrightarrow\left(\mathbb{P}^{\infty}\right)^{n+1}$ is given as the total space of the pull-back of the path-loop fibration $\left(S^{1}\right)^{n+1} \rightarrow P \rightarrow\left(\mathbb{P}^{\infty}\right)^{n+1}$. Write $W_{n+1}$ as the double mapping cylinder

$$
W_{n} \times \mathbb{P} \leftarrow 2 W_{n} \times * \rightarrow\left(\mathbb{P}^{\infty}\right)^{n} \times * .
$$

The fiber of $W_{n} \times \mathbb{P} \rightarrow\left(\mathbb{P}^{\infty}\right)^{n+1}$ is $S^{2 n-1}$ by the induction hypothesis, while the fiber of $\left(\mathbb{P}^{\infty}\right)^{n} \rightarrow\left(\mathbb{P}^{\infty}\right)^{n+1}$ is $\Omega \mathbb{P}=S^{1}$. It follows that the fiber of $W_{n} \times * \simeq$ $W_{n} \times S^{\infty} \rightarrow\left(\mathbb{P}^{\infty}\right)^{n+1}=\left(\mathbb{P}^{n}\right) \times \mathbb{P}$ is $S^{2 n-1} \times S^{1}$, and hence the homotopy fiber $W_{n+1} \mathbb{P} \rightarrow\left(\mathbb{P}^{\infty}\right)^{n+1}$ can be written as the mapping cylinder $S^{2 n-1} \times S^{1} \times[0,1] / \sim$ with $S^{1}$ collapsed at one end and $S^{2 n-1}$ collapsed at the other. But this is no other than $S^{2 n-1} * S^{1}=S^{2 n+1}$, and the proof is complete. 
Corollary 7.3. We have the following commutative diagram of fibrations (here $h$ denotes the Hopf map and $\Delta_{n+1}$ is the diagonal inclusion):

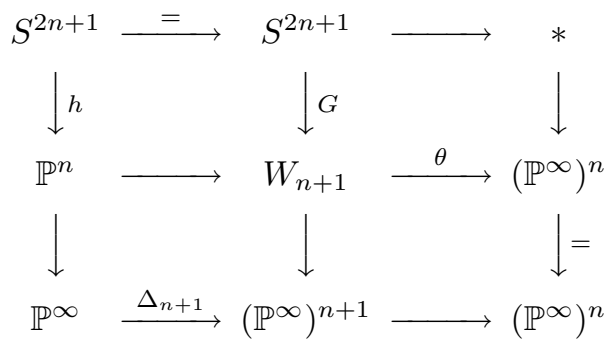

Remark 7.4. Looping 7.2 yields a principal fibration $\Omega(i): \Omega\left(S^{2 n-1}\right) \rightarrow \Omega\left(W_{n}\right) \rightarrow$ $\Omega\left(\mathbb{P}^{\infty}\right)^{n}$ which admits a cross section obtained as follows. Let $s_{i}$ be the inclusion of $\mathbb{P}^{\infty}$ into $W_{n}$ as the $i$-th factor. Then the composition

$$
s:\left(\Omega \mathbb{P}^{\infty}\right)^{n} \stackrel{\left(\Omega s_{1} \times \cdots \times \Omega s_{n}\right)}{\longrightarrow}\left(\Omega W_{n}\right)^{n} \stackrel{*}{\longrightarrow} \Omega W_{n}
$$

provides the desired section of 7.2 (here $*$ is loop multiplication). Naturally this implies that

$$
\Omega W_{n} \simeq\left(\Omega \mathbb{P}^{\infty}\right)^{n} \times \Omega\left(S^{2 n-1}\right) \simeq\left(S^{1}\right)^{n} \times \Omega\left(S^{2 n-1}\right) .
$$

This splitting is not an $H$-space splitting (in the case $n=2$ for instance, the right hand side is abelian while $\Omega(\mathbb{P} \vee \mathbb{P})$ is not). The inclusion $\Omega S^{2 n-1} \hookrightarrow \Omega W_{n}$ is, however, loop-sum preserving.

Lemma 7.5. Consider 7.2; $S^{2 n-1} \stackrel{G}{\rightarrow} W_{n} \hookrightarrow\left(\mathbb{P}^{\infty}\right)^{n}$, and let $a_{i}$ denote the homotopy class of the ith inclusion $S^{2}=\mathbb{P}^{1} \hookrightarrow 1^{i-1} \times \mathbb{P}^{1} \times 1^{n-i} \hookrightarrow\left(\mathbb{P}^{\infty}\right)^{n}$. Then $G$ is an iterated Whitehead product

$$
\left.G=\left[\cdots\left[\left[a_{1}, a_{2}\right], a_{3}\right], \ldots\right], a_{n}\right] .
$$

Remark 7.6. One can apply the functor $\operatorname{Map}^{*}\left(M_{g},-\right)$ to 7.3 and obtain a new diagram of fibrations. It is not hard to see that $\operatorname{Map}_{c}^{*}\left(M_{g}, \mathbb{P}^{\infty}\right) \simeq\left(S^{1}\right)^{2 g}$, where Map $_{c}^{*}$ is any component of Map*. Indeed, since the attaching map of the two disc in $M_{g}$ maps into $\bigvee^{2 g} S^{1}$ as a commutator, it follows that its suspension is null. This implies that

$$
\begin{aligned}
\operatorname{Map}^{*}\left(M_{g}, \mathbb{P}^{\infty}\right) & \simeq \operatorname{Map}^{*}\left(\Sigma M_{g}, K(\mathbb{Z}, 3)\right) \simeq \operatorname{Map}^{*}\left(S^{3} \vee \bigvee S^{2 g}, K(\mathbb{Z}, 3)\right) \\
& \simeq \mathbb{Z} \times \coprod^{2 g} \Omega^{2}(K(\mathbb{Z}, 3)) \simeq \mathbb{Z} \times\left(S^{1}\right)^{2 g}
\end{aligned}
$$

One therefore gets the diagram

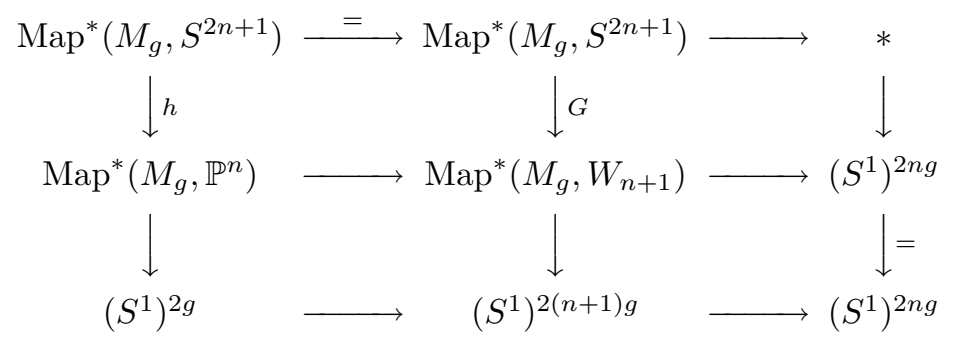


When $n=1$, we know that

$$
\operatorname{Map}^{*}\left(M_{g}, S^{3}\right) \simeq\left(\Omega^{2} S^{3}\right) \times\left(\Omega S^{3}\right)^{2 g}
$$

and that $\operatorname{Map}^{*}\left(M_{g}, S^{2}\right)$ splits as $\left(\Omega S^{3}\right)^{2 g} \times X_{g}$ for some total space $\Omega^{2}\left(S^{3}\right) \rightarrow X_{g} \rightarrow$ $\left(S^{1}\right)^{2 g}$ (see [BCM], $\{11$; or [C2M2], $\{7$ ). This however still is not enough to conclude any splitting for $\operatorname{Map}^{*}\left(M_{g}, \mathbb{P}^{\infty} \vee \mathbb{P}^{\infty}\right)$ (see $\left.\S 8.2\right)$.

The Samelson Product. This is standard $[\mathrm{C}]$ but we review it briefly. Given a loop space $\Omega(X)$, we denote by $S: \Omega(X) \times \Omega(X) \rightarrow \Omega(X)$ the commutator map $S(f, g)=f * g * f^{-1} * g^{-1}$. The map $S$ is null homotopic when either $f$ or $g$ is constant at the basepoint, and hence it descends to a map $S: \Omega(X) \wedge \Omega(X) \rightarrow \Omega(X)$. The Samelson product

$$
\langle,\rangle: \pi_{p}(\Omega(X)) \otimes \pi_{q}(\Omega(X)) \rightarrow \pi_{p+q}(\Omega(X))
$$

is defined to be the composite $S^{p} \wedge S^{q} \stackrel{\alpha \wedge \beta}{\longrightarrow} \Omega(X) \wedge \Omega(X) \stackrel{S}{\longrightarrow} \Omega(X)$.

Theorem 7.7 (Samelson). Consider the suspension $E: X \rightarrow \Omega \Sigma X$ and the induced map ad $: X \wedge X \rightarrow \Omega \Sigma X$ given by $S \circ(E \wedge E)$. Then if $x$ and $y$ are primitive, we have

$$
a d_{*}(x \otimes y)=x \otimes y-(-)^{|x||y|} y \otimes x \in H_{*}(\Omega \Sigma X) \cong T\left(H_{*}(X)\right) .
$$

7.2. Segal's Scanning Map. We can now describe the map

$$
S: \operatorname{Div}_{k}^{n}\left(M_{g}-*\right) \rightarrow \operatorname{Map}_{\overrightarrow{0}}^{*}\left(M_{g}, W_{n} \mathbb{P}\right)
$$

where $\operatorname{Map}_{\overrightarrow{0}}^{*}\left(M_{g}, W_{n} \mathbb{P}\right)$ refers to the subspace of based, null-homotopic maps (or equivalently-based maps of multidegree $\overrightarrow{0}=(0, \ldots, 0))$ in $\operatorname{Map}_{\overrightarrow{0}}\left(M_{g}, W_{n}\right) \subset$ $\operatorname{Map}_{\overrightarrow{0}}\left(M_{g},\left(\mathbb{P}^{\infty}\right)^{n}\right)$.

Fix $r>0(r$ small $)$ and let $D_{r}(x) \subset M_{g}$ be the disc of radius $r$ around the point $x \in M_{g}$. Since $M_{g}-*$ is parallelizable, one can canonically identify the pair $(\bar{D}(x), \partial \bar{D}(x))$ with $\left(S^{2}, \infty\right)$, where the north pole $\infty$ is chosen to be the basepoint in $S^{2}$. To a given positive divisor $D \in S P^{r}\left(M_{g}\right)$ and to any $x \in M_{g}$, we can associate the divisor $D^{x} \in S P^{\infty}\left(S^{2}, \infty\right)=\mathbb{P}$ made out of points of $D \cap D_{r}(x)$ and extended out by basepoints; i.e.

$$
D^{x}=\left\langle D \cup D_{r}(x), \infty, \ldots\right\rangle .
$$

Let $\left(D_{1}, \ldots, D_{n}\right) \in \operatorname{Div}_{k}^{n}\left(M_{g}-*\right)$; then one defines

$$
S: M_{g} \rightarrow \operatorname{Div}^{n}\left(S^{2}, \infty\right), x \mapsto\left(D_{1}^{x}, \ldots, D_{n}^{x}\right)
$$

where here $\operatorname{Div}^{n}\left(S^{2}, \infty\right) \subset\left(\mathbb{P}^{\infty}\right)^{n}$ consists of all $n$-tuples of divisors whose supports do not have a point in common (here the support of $D=\sum n_{i} z_{i} \in S P^{\infty}\left(S^{2}, \infty\right)$ is the set of $\left.z_{i} \neq \infty\right)$. One should probably point out the important difference in topology between $\operatorname{Div}^{n}\left(S^{2}, \infty\right)$ and $\operatorname{Div}^{n}\left(S^{2}-\infty\right)$.

As was observed in [S], we can let $Q_{\epsilon}$ be the open subset of $\operatorname{Div}^{n}\left(S^{2}, \infty\right)$ consisting of $n$-tuples of divisors such that (at least) one such divisor, say $D_{i}$, is disjoint from the closed disk of radius $\epsilon$ about the origin (south pole). Then radial expansion defines a deformation retraction of $Q_{\epsilon}$ into $W_{n}$ (more precisely in this case, the $i$ th component of $\left(\mathbb{P}^{\infty}\right)^{n}$ gets retracted to $\left.\infty\right)$. This shows that $Q_{\epsilon} \simeq W_{n}$, and since $\operatorname{Div}^{n}\left(S^{2}, \infty\right)=\bigcup_{\epsilon>0} Q_{\epsilon}$ we get

Lemma 7.10. $\operatorname{Div}^{n}\left(S^{2}, \infty\right) \simeq W_{n} \mathbb{P}$. 
It is clear that 7.9 has multidegree $(k, \ldots, k)$, and hence when combined with 7.10 it yields a map $S: \operatorname{Div}_{k}^{n}\left(M_{g}-*\right) \rightarrow \operatorname{Map}_{(k, \ldots, k)}^{*}\left(M_{g}, W_{n} \mathbb{P}\right)$. Since all components of the mapping space are homotopy equivalent, we obtain the map 7.8. Note that the stabilization process of $\S 3$ yields a (homotopy) commutative diagram

$$
\begin{array}{ccc}
\operatorname{Div}_{k}^{n}\left(M_{g}-*\right) & \rightarrow & \operatorname{Map}_{\vec{k}}^{*}\left(M_{g}, W_{n} \mathbb{P}\right) \\
\downarrow & & \downarrow \\
\operatorname{Div}_{k+1}^{n}\left(M_{g}-*\right) & \rightarrow & \operatorname{Map}_{k \overrightarrow{+} 1}^{*}\left(M_{g}, W_{n} \mathbb{P}\right),
\end{array}
$$

and in the direct limit we obtain

Theorem 7.11 (Segal). $S: \operatorname{Div}^{n}\left(M_{g}-*\right) \rightarrow \operatorname{Map}_{0}^{*}\left(M_{g}, W_{n} \mathbb{P}\right)$ is a homotopy equivalence.

\section{The Splitting}

Recall that associated to $M_{g}$, we have the cofibration sequence

$$
S^{1} \stackrel{f}{\longrightarrow} \bigvee_{1}^{2 g} S^{1} \longrightarrow M_{g} \longrightarrow S^{2} \longrightarrow \bigvee_{1}^{2 g} S^{2},
$$

with $f$ given as a product of commutators

$$
\left[x_{1}, x_{2}\right] \cdots\left[x_{2 g-1}, x_{2 g}\right] \in \pi_{1}\left(\vee_{1}^{2 g} S^{1}\right) .
$$

Applying the functor $\operatorname{Map}^{*}(-, X)$ to 8.1 yields the fibration sequence

$$
\Omega^{2} X \rightarrow \operatorname{Map}^{*}\left(M_{g}, X\right) \rightarrow(\Omega X)^{2 g} \stackrel{f^{!}}{\longrightarrow} \Omega X .
$$

The map $f^{!}$classifies $\Omega^{2} X \rightarrow \operatorname{Map}^{*}\left(M_{g}, X\right) \rightarrow(\Omega X)^{2 g}$, and since $f^{!}=\left[x_{1}, x_{2}\right]^{!} \cdots$ $\left[x_{2 g-1}, x_{2 g}\right]^{!}$is described in terms of commutators, it is natural to suspect that obstructions to the nullity of $f^{!}$lie in various Whitehead products. This is indeed the case.

Write $X=W_{n}$. To analyze $f^{!}$, it is enough to consider one commutator at a time, say $\left[x_{1}, x_{2}\right]^{!}$. This we write as composition

$$
\left(\Omega W_{n}\right)^{2} \stackrel{\Delta}{\longrightarrow}\left(\left(\Omega W_{n}\right)^{2}\right)^{2} \stackrel{i d^{2} \times \chi^{2}}{\longrightarrow}\left(\left(\Omega W_{n}\right)^{2}\right)^{2} \longrightarrow\left(\Omega W_{n}\right)^{4} \stackrel{*^{4}}{\longrightarrow} \Omega W_{n},
$$

where $\chi$ is the inverse map with respect to the loop sum, $\chi(f)(t)=f(1-t)=f^{-1}(t)$.

In $\S 7$ we saw that we had a map $\Omega W_{n} \stackrel{\pi}{\rightarrow}\left(S^{1}\right)^{n}$ (and a splitting $\Omega W_{n} \simeq \Omega S^{2 n-1} \times$ $\left.\left(S^{1}\right)^{n}\right)$. The composite

$$
\left(\Omega W_{n}\right)^{2} \stackrel{\left[x_{1}, x_{2}\right]^{!}}{\longrightarrow} \Omega W_{n} \stackrel{\pi}{\longrightarrow}\left(S^{1}\right)^{n}
$$

is a commutator in an abelian group and hence it is trivial. It follows that $\pi f^{!}$is also homotopy trivial. and hence $f^{!}$factors (up to homotopy):

$$
f^{!}:\left(\Omega S^{2 n-1} \times\left(S^{1}\right)^{n}\right)^{2 g} \rightarrow \Omega S^{2 n-1} \hookrightarrow \Omega W_{n} .
$$

Question. Does $f^{!}$factor further through $\left(S^{1}\right)^{2 n g}$ as

$$
f^{!}:\left(\Omega S^{2 n-1} \times\left(S^{1}\right)^{n}\right)^{2 g} \rightarrow\left(S^{1}\right)^{2 n g} \rightarrow \Omega S^{2 n-1} \hookrightarrow \Omega W_{n} .
$$

In studying $\operatorname{Div}_{k}^{2}\left(M_{g}-*\right)$, [C2M2] only needed to consider the case $n=2$, and the question above was conjectured to be true.

We can analyze the obstruction to factoring $f^{!}$as in 8.2 as follows. Start with

$$
\left(\Omega S^{2 n-1} \times\left(S^{1}\right)\right)^{2} \stackrel{(\Omega G * e)^{2}}{\longrightarrow} \Omega\left(W_{n}\right)^{2} \stackrel{\left[x_{1}, x_{2}\right]^{!}}{\longrightarrow} \Omega\left(W_{n}\right),
$$


where $e: S^{1} \rightarrow W_{n}$ is any one of the $\Omega s_{i}$ described in 7.5. Letting $e_{1}$ and $e_{2}$ (resp. $\Omega G_{1}$ and $\Omega G_{2}$ ) be the maps of $S^{1}$ (resp. $\Omega S^{2 n-1}$ ) into the first and second copies of $\Omega W_{n}$, one can write (up to sign)

$$
\begin{aligned}
& {\left[x_{1}, x_{2}\right]^{1}\left(\left(e_{1} * \Omega G_{1}\right) \times\left(e_{2} * \Omega G_{2}\right)\right)} \\
& \quad \mapsto e_{1} * \Omega G_{1} * e_{2} * \Omega G_{2} * \chi\left(\Omega G_{1}\right) * \chi\left(e_{1}\right) * \chi\left(\Omega G_{2}\right) * \chi\left(e_{2}\right) .
\end{aligned}
$$

Suppose the image of $\Omega G$ and the image of $e$ commute. Then we can rewrite the above as follows:

$$
\begin{aligned}
\left(e_{1}, e_{2}, \Omega G_{1}, \Omega G_{2}\right) & \mapsto\left(e_{1} * e_{2} *\left[\Omega G_{1} * \Omega G_{2} * \chi\left(\Omega G_{1}\right) * \chi\left(\Omega G_{2}\right)\right] * \chi\left(e_{1}\right) * \chi\left(e_{2}\right)\right) \\
& =e_{1} * e_{2} *\left\{\Omega G_{1}, \Omega G_{2}\right\} * e_{1}^{-1} * e_{2}^{-1} .
\end{aligned}
$$

If we suppose further that $\Omega G_{1}$ and $\Omega G_{2}$ commute in $\Omega W_{n}$, then $f^{!}$would factor as desired through $\left(S^{1}\right)^{2 g n}$. This then shows that the desired factorization 8.2 happens under the following conditions:

- $\Omega G$ and $e$ commute in $\Omega W_{n}$,

- $\Omega S^{2 n-1}$ is homotopy abelian.

The second condition is true after inverting 2. Indeed, an odd sphere is an $\mathrm{H}$ space after inverting 2 , at which point the loop space becomes abelian. To address the validity of the first condition, we restrict our attention to the commutator

$$
\Omega S^{2 n-1} \times S^{1} \stackrel{\{\Omega G, e\}}{\longrightarrow} \Omega\left(W_{n} \mathbb{P}\right) .
$$

Observe that $\Omega S^{2 n-1}=\Omega \Sigma\left(S^{2 n-2}\right) \simeq J\left(S^{2 n-2}\right)$ where $J\left(S^{2 n-2}\right)$ is the James construction on $S^{2 n-2}$ corresponding to the free monoid generated by points of $S^{2 n-2}$. The commutator map can therefore be reduced to $S^{2 n-2} \times S^{1} \stackrel{\{\Omega G, e\}}{\longrightarrow} \Omega\left(W_{n} \mathbb{P}\right)$ and from there one can use the correspondence between the Samelson and Whitehead products $[\mathrm{C}]$ to write

$$
a d\{\Omega G, e\}=[G, a] \in \pi_{2 n}\left(W_{n} \mathbb{P}\right)=\pi_{2 n} S^{2 n-2}=\mathbb{Z}_{2},
$$

where $a=$ ade $: \Sigma S^{1}=S^{2} \hookrightarrow \mathbb{P} \hookrightarrow W_{n}$.

In either case, then, it follows that the obstructions to factoring $f^{!}:\left(W_{n} \mathbb{P}\right)^{2 g} \rightarrow$ $\Omega S^{2 n-1} \times\left(S^{1}\right)^{n}$ through $\left(S^{1}\right)^{n}$ are $\mathbb{Z}_{2}$ obstructions. We have proved the following.

Proposition 8.4. The following splits after inverting 2:

$$
\operatorname{Map}^{*}\left(M_{g}, W_{n} \mathbb{P}\right) \simeq(\mathbb{Z})^{n} \times \Omega\left(S^{2 n-1}\right)^{2 g} \times Y_{g, n},
$$

where $Y_{g, n}$ is the total space of a (principal) fibering $\Omega^{2}\left(S^{2 n-1}\right) \rightarrow Y_{g, n} \rightarrow\left(S^{1}\right)^{2 g n}$.

8.1. The obstruction when $n=2$. When $n>2, n \neq 4,8$, it is clear that 8.4 is best possible. However when $n=2$, one can hope to relax the localization condition there, for in this case $\Omega S^{3}$ is homotopy abelian ( $S^{3}$ being a group) and the first obstruction discussed earlier is not essential. We show, however, that the second obstruction 8.3 is.

Let $G$ be as in Lemma 7.5. We know that the homotopy class of $G$ is represented by $\left[a_{1}, a_{2}\right]$ and hence $\left[G, a_{1}\right]$ corresponds to $\left[\left[a_{1}, a_{2}\right], a_{1}\right]$. We show that this triple Whitehead product generates $\pi_{4}(\mathbb{P} \vee \mathbb{P}) \cong \mathbb{Z}_{2}$.

We start by considering the first few stages of the Postnikov decomposition for $X=\mathbb{P} \vee \mathbb{P}$. Notice that

$$
\pi_{1}(X)=0, \pi_{2}(X) \cong \mathbb{Z} \times \mathbb{Z}, \pi_{3}(X) \cong \mathbb{Z} \text { and } \pi_{4}(X) \cong \mathbb{Z}_{2} .
$$


and hence

$$
\begin{array}{ccccc}
K\left(\mathbb{Z}_{2}, 4\right) & \rightarrow & X_{4} & & \\
& & \downarrow & & \\
K(\mathbb{Z}, 3) & \stackrel{i}{\rightarrow} & X_{3} & \stackrel{k^{5}}{\rightarrow} & K\left(\mathbb{Z}_{2}, 5\right) \\
& & \downarrow & & \\
\mathbb{P} \vee \mathbb{P} & \stackrel{f_{2}}{\rightarrow} & \mathbb{P} \times \mathbb{P} & \stackrel{k^{4}}{\rightarrow} & K(\mathbb{Z}, 4),
\end{array}
$$

where $f_{2}$ is the inclusion $\mathbb{P} \vee \mathbb{P} \hookrightarrow \mathbb{P} \times \mathbb{P}$. The fiber of $f_{2}$ is $S^{3}$, and so

$$
\tau\left(\iota_{3}\right)=k^{4}, \iota_{3} \in \mathbb{Z} \cong H^{3}\left(S^{3}, \pi_{3}(X)\right),
$$

where $\tau$ is the transgression. Since $H^{*}(\mathbb{P} \times \mathbb{P}) \cong \mathbb{Z}\left[a_{1}, a_{2}\right]$, with $a_{1}$ and $a_{2}$ being the dual cohomology classes to the 2-dimensional generators corresponding to the inclusions $S^{2} \hookrightarrow \mathbb{P} \hookrightarrow \mathbb{P} \times \mathbb{P}$, and since

$$
H^{*}(\mathbb{P} \vee \mathbb{P}) \cong \mathbb{Z}\left[a_{1}, a_{2}\right] /\left(a_{1} a_{2}\right),
$$

it follows that the class $a_{1} a_{2}$ must be hit by the transgression and hence $k^{4}=a_{1} a_{2}$.

Lemma 8.5. Let $\gamma$ be the class in $H^{5}\left(X_{3}, \mathbb{Z}_{2}\right)$ that restricts to $S q^{2}\left(\iota_{3}\right), \iota_{3} \in$ $H^{3}\left(K(\mathbb{Z}, 3), \mathbb{Z}_{2}\right)$. Then $\gamma$ is non-zero, and $k^{5}=\gamma$.

Proof. Since $d_{4}$ corresponds to the transgression in this case, we have $d_{4}\left(\iota_{3}\right)=$ $\tau\left(\iota_{3}\right)=a_{1} a_{2}$. Recall that

$$
H^{*}\left(K(\mathbb{Z}, 3), \mathbb{Z}_{2}\right)=\mathbb{F}_{2}\left[\iota_{3}, S q^{2}\left(\iota_{3}\right),\left(\iota_{3}\right)^{2}, S q^{4} S q^{2}\left(\iota_{3}\right), \ldots, S q^{2^{i}} \cdots S q^{4} S q^{2}\left(\iota_{3}\right), \ldots\right] .
$$

A quick inspection of the $E_{4}$ quadrant shows that the $d_{4}$ differential vanishes on all homology generators in the fiber but $\iota_{3}$. Since $d_{4}\left(\iota_{3}\right)=a_{1} a_{2}$, and since the classes $a_{1}$ and $a_{2}$ survive (and their powers), it follows that

$$
E_{5}=H^{*}(\mathbb{P} \times \mathbb{P}) \otimes \mathbb{F}_{2}\left[S q^{2}\left(\iota_{3}\right), \iota_{3}^{2}, S q^{4} S q^{2}\left(\iota_{3}\right), \ldots\right] .
$$

Since $\iota_{3}$ transgresses, so does $S q^{2}\left(\iota_{3}\right)$. We then have

$$
d_{6}\left(S q^{2}\left(\iota_{3}\right)\right)=S q^{2}\left(d_{4}\left(\iota_{3}\right)\right)=S q^{2}\left(a_{1} a_{2}\right) .
$$

But $S q^{2}\left(a_{1} a_{2}\right)$ is already hit by $d_{4}$, as the following application of the Cartan formula (with $S q^{1}\left(a_{i}\right)=0$ in $\mathbb{P}$ ) shows:

$$
S q^{2}\left(a_{1} a_{2}\right)=S q^{2}\left(a_{1}\right) a_{2}+a_{1} S q^{2}\left(a_{2}\right)=\left(a_{1}+a_{2}\right) a_{1} a_{2}=d_{4}\left(a_{1}+a_{2}\right) \iota_{3} .
$$

It follows that $d_{6}\left(S q^{2}\left(\iota_{3}\right)\right)=0$ and that $S q^{2}\left(\iota_{3}\right)$ survives to $E_{\infty}$. Since it is the only class in $H^{5}\left(X_{3}, \mathbb{Z}_{2}\right)$, it must be the image of the transgression $\tau\left(H^{4}\left(K\left(\mathbb{Z}_{2}, 4\right), \mathbb{Z}_{2}\right)\right)$ in the next stage of the Postnikov tower. This proves the lemma.

Now consider the pulback diagram

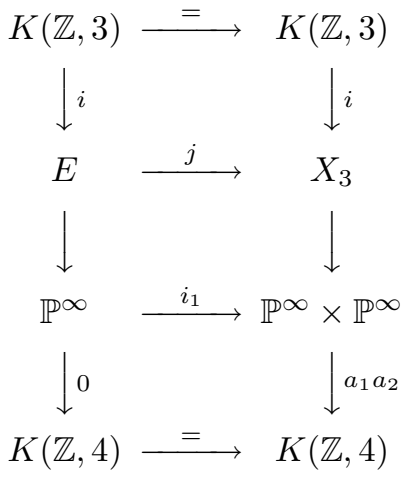


where $i_{1}$ is the inclusion of $\mathbb{P}$ into the first factor. The pullback of the $k$-invariant $a_{1} a_{2}$ under $i_{1}$ is trivial (since $i_{1}^{*}\left(a_{2}\right)=0$ ). The induced total space is then $E=$ $K(\mathbb{Z}, 3) \times K(\mathbb{Z}, 2)$

Lemma 8.6. $j^{*}(\gamma)=S q^{2}\left(\iota_{3}\right)+\iota_{3} \cup a_{1}$.

Proof. In the fibration

$$
K(\mathbb{Z}, 3) \stackrel{i}{\rightarrow} X_{3} \stackrel{p}{\rightarrow} \mathbb{P} \times \mathbb{P}
$$

$\tau\left(S q^{2}\left(\iota_{3}\right)\right)=a_{1} a_{2}\left(a_{1}+a_{2}\right)$ means that there is a class $\beta \in \mathcal{C}^{5}\left(X_{3}\right)$ (where $(\mathcal{C}, \delta)$ is a cochain complex) such that $i^{*}([\beta])=S q^{2}\left(\iota_{3}\right)$ (this is the only time that we differentiate between a cochain $x$ and its cohomology class $[x])$ and that

$$
\delta(\beta)=p^{*}\left(a_{1} a_{2}\left(a_{1}+a_{2}\right)\right)=p^{*}\left(a_{1} a_{2}\right) \cup p^{*}\left(a_{1}+a_{2}\right) .
$$

The cochain $\beta$ is chosen modulo $p^{*}(\mathbb{P} \vee \mathbb{P})$. Now, the pullback $i^{*}\left(j^{*}(\beta)\right)$ must correspond to $S q^{2}\left(\iota_{3}\right)$ and hence $j^{*}(\beta)-S q^{2}\left(\iota_{3}\right) \in \operatorname{ker}\left(i^{*}\right)=\left\{0, i_{3} \cup a_{1}\right\}$. By the choice of $\beta$ modulo $p^{*}(\mathbb{P} \vee \mathbb{P})$, we must then have that $j^{*}(\beta)=S q^{2}\left(\iota_{3}\right)$.

On the other hand, since $\iota_{3}$ transgresses to $a_{1} a_{2}$ it follows also that $p^{*}\left(a_{1} a_{2}\right)=$ $\delta\left(\iota_{3}\right)$. (Here we think of $\iota_{3}$ as some cochain in $X_{3}$ mapping onto $\iota_{3} \in H^{3}(K(\mathbb{Z}, 3)$ ) under the epimorphism $i^{*}: \mathcal{C}^{3}\left(X_{3}\right) \rightarrow \mathcal{C}^{3}(K(\mathbb{Z}, 3))$.) It then follows that

$$
\delta(\beta)=\delta\left(\iota_{3}\right) \cup\left(a_{1}+a_{2}\right)=\delta\left(\iota_{3}\left(a_{1}+a_{2}\right)\right)
$$

and hence that $\delta\left(\beta+\iota_{3}\left(a_{1}+a_{2}\right)\right)=0$. Moreover $i^{*}\left(\beta+\iota_{3}\left(a_{1}+a_{2}\right)\right)=S q^{2}\left(\iota_{3}\right)$. This shows that we can choose $\gamma \in H^{5}\left(X_{3}\right)$ to be equal to $\beta+\iota_{3}\left(a_{1}+a_{2}\right)$. Therefore

$$
j^{*}(\gamma)=j^{*}(\beta)+j^{*}\left(\iota_{3}\left(a_{1}+a_{2}\right)\right)=S q^{2}\left(\iota_{3}\right)+\iota_{3} \cup a_{1},
$$

and the lemma follows.

Lemma 8.7. $\left[\left[a_{1}, a_{2}\right], a_{1}\right]$ generates $\pi_{4}(\mathbb{P} \vee \mathbb{P}) \cong \mathbb{Z}_{2}$.

Proof. Consider the composite map

$$
S^{3} \times S^{2} \stackrel{f=\iota_{3} \times \iota_{2}}{\longrightarrow} E=K(\mathbb{Z}, 3) \times K(\mathbb{Z}, 2) \stackrel{j}{\longrightarrow} X_{3} .
$$

The pullback of $\gamma$ via $f \circ j$ is

$$
f^{*}\left(j^{*}(\gamma)\right)=f^{*}\left(S q^{2}\left(\iota_{3}\right)\right)+f^{*}\left(\iota_{3} \cup a_{1}\right)=f^{*}\left(\iota_{3}\right) \cup f^{*}\left(a_{1}\right)=\kappa_{3} \cup \kappa_{2},
$$

where $\kappa_{i}$ is the generator of $H^{i}\left(S^{i}\right)$. It follows that the composite $S^{3} \times S^{2} \rightarrow X_{3} \rightarrow$ $K\left(\mathbb{Z}_{2}, 5\right)$ is not zero and hence $S^{3} \times S^{2}$ does not lift to the next stage, $X_{4}$, of the Postnikov resolution, since the latter $K\left(\mathbb{Z}_{2}, 4\right) \rightarrow 1.5 X_{4} \rightarrow 1.5 X_{3}$ has $k$-invariant $\gamma$.

The fact that $S^{3} \times S^{2}$ doesn't lift to $X_{4}$ implies that the class of the Whitehead product $\left[\kappa_{3}, \kappa_{2}\right] \in \pi_{3}\left(S^{3} \vee S^{2}\right)$ has image the non-zero generator in $\pi_{4}\left(E_{2}\right)=$ $\pi_{4}(\mathbb{P} \vee \mathbb{P})$ (here $\kappa_{i}$ also denotes the generating class in $\pi_{i}\left(S^{i}\right)$ ). The image of $\kappa_{2}$ is $a_{1}$ by construction, while the image of $\kappa_{1} \in \pi_{3}\left(S^{3}\right)$ is the Whitehead product $\left[a_{1}, a_{2}\right] \in \pi_{3}(\mathbb{P} \vee \mathbb{P})$ according to the diagram

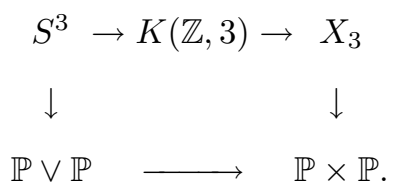

This concludes the proof. Note that the non-zero generator $\left[\left[a_{1}, a_{2}\right], a_{1}\right]$ must correspond to $\left[a_{1}, a_{2}\right] \circ \eta$, where $\eta$ is the Hopf map $S^{4} \rightarrow S^{3}$. 


\section{Relation with Mapping Spaces}

In this section, we prove the following easy consequence of our previous study of the divisor spaces.

Proposition 9.1. The Eilenberg-Moore spectral sequence associated to the fibration

$$
\Omega^{2} S^{2 n-1} \rightarrow \operatorname{Map}_{0}^{*}\left(M_{g}, W_{n} \mathbb{P}\right) \rightarrow\left(S^{1}\right)^{2 n g} \times\left(\Omega S^{2 n-1}\right)^{2 g}
$$

collapses at $E_{2}=\operatorname{Tor}_{H^{*}\left(\Omega S^{2 n-1}\right)}\left(\mathbb{F}, H^{*}\left(\left(S^{1}\right)^{2 n g} \times\left(\Omega S^{2 n-1}\right)^{2 g}\right)\right)$.

Proof. Consider first the case $n=2$. In this case, the classifying map being null homotopic on $\Omega S^{3}$, it follows that the action of $H^{*}\left(\Omega S^{3}\right)$ on $H^{*}\left(\Omega S^{3}\right)^{2 g}$ is trivial and that

$$
E_{2}=H^{*}\left(\Omega S^{3}\right)^{2 g} \otimes \operatorname{Tor}_{H^{*}\left(\Omega S^{3}\right)}\left(\mathbb{F}, H^{*}\left(S^{1}\right)^{4 g}\right) .
$$

We write $H^{*}\left(S^{1}\right)^{4 g}=\Lambda\left(e_{1}, \ldots, e_{4 g}\right)$ and $H^{*}\left(\Omega S^{3}\right)=\Gamma(a)$. We should point out that in the Eilenberg-Moore spectral sequence the bar degrees are subtracted from the total degree of resolution elements rather than added (compare with §1). For instance, in this case $\operatorname{deg}|a|=2-1=1$. To understand the module structure of $\Gamma(a)$ on $\Lambda\left(e_{1}, \ldots, e_{4 g}\right)$, we need to know first about the ring structure of $H_{*}(\Omega(\mathbb{P} \vee \mathbb{P}))$.

Lemma 9.2 $([\mathrm{C} 2 \mathrm{M} 2])$. Let $e_{1}, e_{2} \in H_{1}(\Omega(\mathbb{P} \vee \mathbb{P}))$ be the generators corresponding to the inclusions of $\mathbb{P}$ into the first and second factors of $\mathbb{P} \vee \mathbb{P}$ (respectively), and let a represent the class in the Hurewicz image of the generating sphere in $H_{2}(\Omega(\mathbb{P} \vee \mathbb{P}) ; \mathbb{Z})$ coming from $\pi_{2}\left(\Omega S^{3}\right)$. Then if $T()$ denotes the tensor algebra, we have

$$
H_{*}(\Omega(\mathbb{P} \vee \mathbb{P}) ; \mathbb{Z}) \cong T\left(e_{1}, e_{2}, a\right) /\left(e_{1}^{2}=e_{2}^{2}=0, e_{1} e_{2}+e_{2} e_{1}=a\right) .
$$

Proof. Since $e_{1}, e_{2},\left\langle e_{1}, e_{2}\right\rangle$ and $\left[e_{1}, e_{2}\right]$ are maps of spheres, we will use the same notation for the maps and the corresponding spherical classes they generate. That $e_{1}^{2}=e_{2}^{2}=0$ follows trivially from the homology of $S^{1}$. Since the inclusion $G: S^{3} \rightarrow$ $\mathbb{P} \vee \mathbb{P}$ is given by $G=\left[\Sigma e_{1}, \Sigma e_{2}\right]$ (Lemma 8.5), it then follows that $a=\operatorname{ad} G=$ $\left\langle a d \Sigma e_{1}, a d \Sigma e_{2}\right\rangle=\left\langle e_{1}, e_{2}\right\rangle: S^{2} \rightarrow \Omega S^{3} \rightarrow \Omega(\mathbb{P} \vee \mathbb{P})$. By Samelson's theorem 7.6, we must have that $\left\langle e_{1}, e_{2}\right\rangle=e_{1} e_{2}+e_{2} e_{1}=a$, as desired.

Going back to the proof of 9.1 , we can look at the effect of the commutator $\left[x_{1}, x_{2}\right]^{!}$at the level of homology on $H_{*}\left(S^{1} \times S^{1}\right)$. We have

$$
\begin{aligned}
{\left[x_{1}, x_{2}\right]^{!}\left(e_{i j} \otimes e_{k l}\right) } & =(*) \times\left(i d^{2} \times \chi^{2}\right) \Delta_{*}\left(e_{i j} \otimes e_{k l}\right) \\
& =(*)\left(e_{i j} e_{k l} \otimes 1+e_{i j} \otimes e_{k l}-e_{k l} \otimes e_{i j}+1 \otimes e_{i j} e_{k l}\right)
\end{aligned}
$$

and using the relations in 9.2 above, we see that $\left[x_{1}, x_{2}\right]^{!}\left(e_{i j} \otimes e_{k l}\right)=0$ when $i=k$ or $j=l$, and that

$$
\begin{aligned}
{\left[x_{1}, x_{2}\right]^{!}\left(e_{11} \otimes e_{22}\right) } & =\left[x_{1}, x_{2}\right]^{!}\left(e_{12} \otimes e_{21}\right) \\
& =e_{1} \otimes e_{2}+e_{2} \otimes e_{1}=a \in \mathbb{Z}[a]=H_{*}\left(\Omega S^{3}\right)
\end{aligned}
$$

This then defines the map $f^{!}$completely. In cohomology, it follows that $f^{*}(a)=$ $\sum_{1}^{2 g} e_{2 i+1} e_{2 i}$, which implies that the action of $a$ on $\Lambda\left(e_{1}, e_{2}, \ldots, e_{4 g}\right)$ is given by multiplication with $\sum_{1}^{2 g} e_{2 i+1} e_{2 i}$. In this case $(*)$ takes the form

$$
\Gamma\left(h_{1}, \ldots, h_{2 g}\right) \otimes \operatorname{Tor}_{\Gamma[a]}\left(\mathbb{F}, \Lambda\left(e_{1}, \ldots, e_{4 g}\right)\right) .
$$


This already makes up for the homology $H_{*}\left(\operatorname{Div}^{2}\left(M_{g}-*\right) ; \mathbb{F}\right)(\mathrm{cf} . \S 6.2)$ and hence in light of Segal's homotopy equivalence 7.10 , this must give the entire homology of $\operatorname{Map}_{0}^{*}\left(M_{g}, \mathbb{P} \vee \mathbb{P}\right)$ and $E_{2}=E_{\infty}$.

The case $n>2$ is simpler, for $f^{*}: H^{*}\left(\Omega\left(W_{n} \mathbb{P}\right)\right) \rightarrow H^{*}\left(\left(\Omega W_{n} \mathbb{P}\right)^{2 g}\right)$ is trivial and hence the $E_{2}$ term $\left(^{*}\right)$ takes the form

$$
E_{2}=H^{*}\left(\left(\Omega S^{2 n-1}\right)^{2 g} ; \mathbb{F}\right) \otimes H^{*}\left(\left(S^{1}\right)^{2 n g} ; \mathbb{F}\right) \otimes H^{*}\left(\Omega^{2} S^{2 n-1} ; \mathbb{F}\right) .
$$

Here too results of $\S 6$ show that 9.4 accounts for all classes in $H^{*}\left(\operatorname{Map}_{0}^{*}\left(M_{g}, W_{n}\right) ; \mathbb{F}\right)$, and the Eileberg-Moore spectral sequence must then collapse at $E^{2}$. This completes the proof.

\section{ACKNOWLEDGEMENTS}

This work is part of the author's doctoral dissertation written at Stanford University under the supervision of Professor R.J. Milgram. The author is much indebted to his advisor for suggesting the problem and for sharing ideas without which this work would not have been possible. The author would also like to thank G. Carlsson and R.L. Cohen for helpful conversations. The final version of this paper was written at the Centre de Recherches Mathematiques of the Université of of Montréal, and the author thanks both the center and Professor J. Hurtubise for their support.

\section{REFERENCES}

[ACGH] E. Arbarello, M. Cornalba, P.A. Griffiths, J. Harris, "Geometry of algebraic curves", Springer Grund. Math. Wiss. 267, 1985. MR 96h:14019

[BC] C.F. Bodigheimer, F.R. Cohen, "Rational cohomology of configuration spaces", Proc. Topology Conference, Gottingen (1987), Springer Lect. Notes Math. 1361 (1988), 7-13. MR 90e: 57075

[BCM] C.F. Bodigheimer, F.R. Cohen, R.J. Milgram, "Truncated symmetric products and configuration spaces", Math. Zeit., 214 (1993), 179-216. MR 95a:55043

[Br] Glen E. Bredon, "Equivariant Cohomology Theories", Lecture Notes in Math., vol 34, Springer-Verlag, (1967). MR 35:4914

[Car] H. Cartan, Séminaire Henri Cartan 1954-55, exposés 2-11, Secrétariat Math., Paris, 1955. MR 19:438e

[C] F. Cohen, "A course in some aspects of classical homotopy theory", Springer lecture notes in Math. 1286 (1987), 1-92. MR 89e:55027

[C2M2] F.R. Cohen, R.L. Cohen, B.M. Mann, R.J. Milgram, "The topology of rational functions and divisors of surfaces", Acta Math., 166 (1991), 163-221. MR 92k:55011

[CS] R.L. Cohen, D.H. Shimamoto, "Rational functions, labelled configurations, and Hilbert schemes", J. London. Math. Soc. 43 (1991) 509-528. MR 93c:55009

[D] A. Dold, "Homology of symmetric products and other functors of complexes", Ann. Math. 68 (1958), 54-80. MR 20:3537

[DT] A. Dold, R. Thom, "Quasifaserungen und unendliche symmetrishe Produkte", Ann. Math. 67 (1958), 239-281. MR 20:3542

[Gu] M.A. Guest, "On the space of holomorphic maps from the Riemann sphere to the quadric cone", Quart. J. Math. Oxford Ser. (2) 45 (1994), 57-75. MR 95f:58015

[GH] M.J.Greenberg, J.R.Harper, "Algebraic Topology, A First Course", Addison-Wesley Mathematics Note Series, vol. 58 (1981). MR 83b:55001

[KM] S. Kallel, R.J. Milgram, "The geometry of the space of holomorphic maps from a Riemann sphere to complex projective space", preprint 1995.

[McCl] John McCleary, "User's guide to spectral sequences", Publish or Perish, 1985. MR 87f: 55014

[M1] R.J. Milgram, "The bar construction and abelian H-spaces", Ill. J. Math. 11 (1967), 242250. MR 34:8404

[M2] R.J. Milgram, "The homology of symmetric products", Trans. Amer. Math. Soc. 138 (1969), 251-265. MR 39:3483 
[M3] R.J. Milgram, lecture notes.

[S] G. Segal, "The topology of spaces of rational functions", Acta. Math., 143 (1979), 39-72. MR 81c:55013

[Sp] E. Spanier, "Infinite symmetric products, function spaces, and duality", Ann. Math. 69 (1959), 142-198. MR 21:3851

Department of Mathematics, Stanford University, Stanford, California 94305-2125

Centre de Recherches Mathématiques, Université de Montréal, Montréal, Québec H3C 3J7, CANADA 